\title{
Local and global symbols on compact Lie groups
}

\section{Véronique Fischer $^{1}$ (D)}

Received: 13 January 2019 / Revised: 10 May 2019 / Accepted: 13 May 2019 / Published online: 22 May 2019 (c) The Author(s) 2019

\begin{abstract}
On the torus, it is possible to assign a global symbol to a pseudo-differential operator using Fourier series. In this paper we investigate the relations between the local and global symbols for the operators in the classical Hörmander calculus and describe the principal symbols, the non-commutative residue and the canonical trace of an operator in terms of its global symbol. We also generalise these results to any compact Lie group.
\end{abstract}

Keywords Pseudo-differential operators on manifolds - Analysis on the torus and on compact Lie groups · Non-commutative residue $\cdot$ Canonical trace

Mathematics Subject Classification 58J40 · 43A77 · 58J42

\section{Contents}

1 Introduction . . . . . . . . . . . . . . . . . . . . . . . . 230

2 Preliminaries: The Euclidean case . . . . . . . . . . . . . . . . . . . . . 231

2.1 The Euclidean pseudo-differential calculus . . . . . . . . . . . . . . . . . . 232

2.2 Trace expansions . . . . . . . . . . . . . . . . . . . . 233

2.3 The non-commutative residue . . . . . . . . . . . . . . . . . . . . . . 236

2.4 The canonical trace . . . . . . . . . . . . . . . . . . . . . . . . . . . . . 237

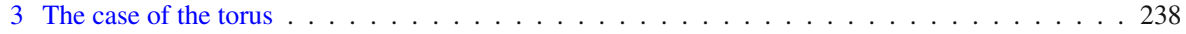

3.1 The toroidal pseudo-differential calculus . . . . . . . . . . . . . . . . 238

3.2 Main results for the classical toroidal pseudo-differential calculus . . . . . . . . . . . . . . 240

3.3 Proofs of Propositions 3.3 and $3.4 \ldots \ldots \ldots$. . . . . . . . . . . . . . . . . . 244

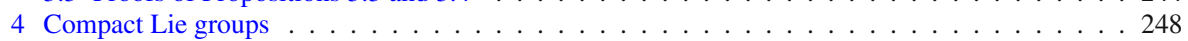

4.1 Notations and conventions $\ldots \ldots \ldots \ldots \ldots$

4.1 .1 Representations . . . . . . . . . . . . . . . . . . . . . . . . . . . . . 248

4.1 .2 Lie algebra and vector fields . . . . . . . . . . . . . . . . . . . . . . . . . 249

4.1 .3 Group Fourier transform . . . . . . . . . . . . . . . . . . . . 250

4.2 The Hörmander pseudo-differential calculus on $G \ldots \ldots \ldots \ldots \ldots$

4.2 .1 The symbols and the natural quantisation . . . . . . . . . . . . . 250

Véronique Fischer

v.c.m.fischer@bath.ac.uk

1 Department of Mathematical Sciences, University of Bath, Claverton Down, Bath BA2 7AY, UK 
4.2 Difference operators . . . . . . . . . . . . . . . . . . . . 251

4.2 .3 Pseudo-differential calculus on $G \ldots \ldots \ldots \ldots$. . . . . . . . . . . 252

4.2 .4 Kernels . . . . . . . . . . . . . . . . . . . . . . . . 253

4.2 .5 Invariance of the calculi under translations . . . . . . . . . . . . . . . . . . . . 254

4.3 Principal symbols . . . . . . . . . . . . . . . . . . . . . . . . 256

4.4 Homogeneous symbols and classical symbols on $G \ldots \ldots$. . . . . . . . . . . . . 258

4.5 Proof of Theorem $4.9 \ldots \ldots \ldots$. . . . . . . . . . . . . . . . . . . 259

4.6 Non-commutative residue and canonical trace on $G \ldots \ldots$. . . . . . . . . . 263

References . . . . . . . . . . . . . . . . . . . . . . . . . . . . 264

\section{Introduction}

On general smooth manifolds, an operator is pseudo-differential when it belongs to some Hörmander class $\Psi^{m}$ in local coordinates: this property is local. Although each operator remains globally defined, one cannot hope to extract global geometric information from its local symbols in coordinate charts. However, in some settings, it is possible to define a notion of global symbols: it is naturally the case for operators on $\mathbb{R}^{n}$ (see e.g. [23]), but also on the torus, starting with the work of Agranovitch in dimension one in 1979 [1], followed by many studies of toroidal or periodical pseudodifferential operators, e.g. [2,21,22,35]. Recently, this idea has been studied on the non-commutative torus by Lévy et al. in [20].

In this paper, we study the case of compact Lie groups which may be viewed as a different direction for a non-commutative generalisation of the torus. Indeed, on the torus and more generally on any compact Lie group, it is possible to use the Fourier series to define the global symbol of a pseudo-differential operator on the torus. The aim of this paper is to characterise the classical Hörmander classes in terms of the global symbols, and then to investigate the relations between the global symbols and the local symbols in coordinate charts. In particular, we will relate the global symbols with local objects such as the principal symbol and the non-commutative residue of an operator, and also with its (global) canonical trace. To our knowledge, these results are new.

The definitions of the non-commutative residue and of the canonical trace together with further references will be given in Sect.2, so in this introduction we will restrict our comments to their origins and uses. The non-commutative residue was introduced independently by Guillemin [12] and Wodzicki [36,37] in the early eighties. Beside being the only trace on the algebra of pseudo-differential operators up to constants, its importance comes from its applications in mathematical physics, mainly in Connes' non-commutative geometry due to its link with the Dixmier trace [3] but also in relation with e.g. the Einstein-Hilbert action (see [19, Section 6.1] and the references therein). The canonical trace was constructed by Kontsevich and Vishik in the mid-nineties [17] as a tool to study further zeta functions and determinants of elliptic pseudodifferential operators. Since then, it has received considerable attention and found interesting applications, see e.g. [24,25,30].

On the torus $\mathbb{T}^{n}$, using trigonometric Fourier series, the relations between local and global symbols are well understood [1,2,21,22,27,35]: a global symbol $\sigma$ is defined on $\mathbb{T}^{n} \times \mathbb{Z}^{n}$, and, when the corresponding operator is classical, this global symbol 
extends naturally to a symbol on $\mathbb{T}^{n} \times \mathbb{R}^{n}$ which coincides with the local symbol modulo smoothing symbols, see Sect. 3 . Therefore the notions of principal symbol and non-commutative residue have obvious meanings locally and globally. Furthermore, the recent result of the author of this paper on real trace expansions [6] applied to the Laplacian on the torus yields a description of the non-commutative residue and of the canonical trace as coefficients in certains expansions. This generalises Pietsch's result on the non-commutative residue on the torus in [26, Theorem 11.15]. It also gives an alternative proof of the description of the canonical trace as the discrete Hadamard finite part of the symbol already obtained by Lévy et al. in [20, Section 5].

The case of compact Lie groups is more involved but also more natural from the viewpoint of harmonic analysis. Indeed, the idea of global symbols stems from the study of Fourier multipliers, and is related to singular integrals (Caledròn-Zygmund theory) and to the genesis of the pseudo-differential theory; see [7] and its introduction for a brief survey on Fourier multipliers on compact Lie groups in these directions. The idea of studying pseudo-differential operators on Lie groups as a systematic generalisation of Fourier multipliers can be traced back to Michael Taylor in [34]. On compact Lie groups, recent works $[8,27,28]$ have shown that it is possible to characterise the classes of global symbols corresponding to pseudo-differential operators (see Sect. 4.2). In this paper, we also characterise the classical pseudo-differential classes and show how to obtain the principal symbol from the global symbol (see Sects. 4.3 and 4.4); these results are new to our knowledge. They use representation theory, especially highest weights. They recover the commutative case of the torus with a very sophisticated presentation.

The paper is organised as follows. In Sect. 2, we review the properties of the classical pseudo-differential calculus on any compact manifold. In particular, we recall the notions of principal symbols, non-commutative residue and canonical trace. We explore the case of the torus in Sect. 3, the main result in the toroidal setting being summarised in Sect.3.2. In Sect.4, we examine the case of compact Lie groups: we first relate the principal symbol of a classical operator with the global symbol in Sect. 4.3. Subsequently, in Sect.4.4, we define the notions of global homogeneous symbol and global classical symbols, and characterise classical pseudo-differential operators as the pseudo-differential operators with classical symbols. We conclude with the derivation of the non-commutative residue and the canonical trace as coefficients in certains expansions.

\section{Preliminaries: The Euclidean case}

In this section we recall well-known properties of the classical Hörmander pseudodifferential calculus on $\mathbb{R}^{n}$ and on a manifold. We also introduce the non-commutative residue and the canonical trace together with their relations to trace expansions. 


\subsection{The Euclidean pseudo-differential calculus}

In this section we mainly set the notation and our vocabulary for the pseudo-differential calculus on an open subset $\Omega$ of $\mathbb{R}^{n}$ and on a compact manifold $M$. Classical references for this material include $[32,33]$.

We denote by $S^{m}=S^{m}(\Omega)$ the Hörmander class of symbols of order $m \in \mathbb{R}$ on $\Omega$, that is, the Fréchet space of smooth functions $a: \Omega \times \mathbb{R}^{n} \rightarrow \mathbb{C}$ satisfying for all multi-indices $\alpha, \beta \in \mathbb{N}_{0}^{n}$

$$
\left|\partial_{x}^{\beta} \partial_{\xi}^{\alpha} a(x, \xi)\right| \leq C_{\alpha, \beta}\langle\xi\rangle^{m-\alpha}
$$

In this paper, we will regularly use the usual notation $\langle\xi\rangle=\sqrt{1+|\xi|^{2}}, \partial_{j}=\partial_{x_{j}}$ for the partial derivatives in $\mathbb{R}^{n}, \partial^{\alpha}=\partial_{1}^{\alpha_{1}} \partial_{2}^{\alpha_{2}} \ldots$ etc. $\mathbb{N}_{0}=\{0,1,2, \ldots\}$ denotes the set of non-negative integers and $\mathbb{N}=\{1,2, \ldots\}$ the set of positive integers.

We say that the symbol $a \in S^{m}(\Omega)$ is compactly supported in $x$ when there exists $R>0$ such that $a(x, \xi)=0$ for any $(x, \xi) \in \mathbb{R}^{n} \times \mathbb{R}^{n}$ with $|x|>M$.

To each symbol $a \in S^{m}(\Omega)$, we associate the operator $\operatorname{Op}_{\Omega}(a)$ defined via

$$
\mathrm{Op}_{\Omega}(a) f(x)=\int_{\mathbb{R}^{n}} \widehat{f}(\xi) e^{2 i \pi x \cdot \xi} a(x, \xi) d \xi, \quad x \in \Omega, f \in C_{c}^{\infty}(\Omega) .
$$

Here, $\widehat{f}$ denotes the Euclidean Fourier transform of $f \in \mathcal{S}\left(\mathbb{R}^{n}\right)$ :

$$
\widehat{f}(\xi)=\mathcal{F}_{\mathbb{R}^{n}} f(\xi)=\int_{\mathbb{R}^{n}} f(x) e^{-2 i \pi x \cdot \xi} d x
$$

We denote by $\Psi^{m}=\Psi^{m}(\Omega)=\mathrm{Op}_{\Omega}\left(S^{m}(\Omega)\right)$ the Hörmander class of operators of order $m \in \mathbb{R}$ on $\Omega$. Recall that $\mathrm{Op}_{\Omega}$ is one-to-one on $S^{m}(\Omega)$ and thus that $\Psi^{m}(\Omega)$ inherit a structure of Fréchet space.

The class of smoothing symbols is denoted by $S^{-\infty}=S^{-\infty}(\Omega)=\cap_{m \in \mathbb{R}} S^{m}(\Omega)$ and the class of smoothing symbols is denoted by $\Psi^{-\infty}(\Omega)=\cap_{m \in \mathbb{R}} \Psi^{m}(\Omega)=$ $\mathrm{Op}_{\Omega}\left(S^{-\infty}(\Omega)\right)$. Examples of smoothing operators are convolution operators with Schwartz convolution kernels.

If $A \in \cup_{m \in \mathbb{R}} \Psi^{m}(\Omega)$ then we denote by $K_{A} \in \mathcal{S}^{\prime}(\Omega \times \Omega)$ its integral kernel so that we have in the sense of distributions:

$$
A f(x)=\int_{\Omega} K_{A}(x, y) f(y) d y .
$$

Recall that $a(x, \xi)=\mathcal{F}_{\mathbb{R}^{n}}\left(K_{A}(x, x-\cdot)\right)$ and that $K_{A}$ is smooth away from the diagonal $x=y$.

The following property is well-known:

Lemma 2.1 If $A=\mathrm{Op}_{\Omega}(a) \in \Psi^{m}(\Omega)$ with $m<-n$, then its integral kernel $K_{A}$ is continuous. Assuming furthermore that its symbol $a \in S^{m}(\Omega)$ is compactly supported in $x$, then the operator $A$ is trace-class with trace: 


$$
\operatorname{tr}(A)=\int_{\Omega} K_{A}(x, x) d x=\int_{\Omega \times \mathbb{R}^{n}} a(x, \xi) d x d \xi .
$$

Consequently, if $\Omega$ is a bounded open subset of $\mathbb{R}^{n}$, then the linear map $A \mapsto \operatorname{tr}(A)$ is continuous on $\Psi^{m}(\Omega)$ for any $m<-n$.

A symbol $a \in S^{m}$ with $m \in \mathbb{R}$ admits a poly-homogeneous expansion with complex order $\tilde{m}$ when it admits an expansion $a \sim \sum_{j \in \mathbb{N}_{0}} \alpha_{m-j}$ where each function $\alpha_{m-j}(x, \xi)$ is in $S^{m-j}$ and is $(\tilde{m}-j)$-homogeneous in $\xi$ for $|\xi| \geq 1$; here $\tilde{m} \in \mathbb{C}$ with $\Re \tilde{m}=m$ and the homogeneity for $|\xi| \geq 1$ means that $\alpha_{m-j}(x, \xi)=|\xi|^{\tilde{m}-j} \alpha_{m-j}(x, \xi /|\xi|)$ for any $(x, \xi) \in \mathbb{R}^{n} \times \mathbb{R}^{n}$ with $|\xi| \geq 1$. We write the poly-homogeneous expansion as $a \sim_{h} \sum_{j} a_{\tilde{m}-j}$ where $a_{\tilde{m}-j}(x, \xi)=$ $|\xi|^{\tilde{m}-j} \alpha_{m-j}(x, \xi /|\xi|) \in C^{\infty}\left(\Omega \times\left(\mathbb{R}^{n} \backslash\{0\}\right)\right)$ is homogeneous of degree $\tilde{m}-j$ in $\xi$. We may call $a_{\tilde{m}}$ the (homogeneous) principal symbol of $a$ or of $A$ and $\tilde{m}$ the complex order of $a$ or $A$.

If the open set $\Omega$ is bounded, we say that a symbol in $S^{m}(\Omega)$ is classical with complex order $\tilde{m}$ when it admits a poly-homogeneous expansion with complex order $\tilde{m}$. We denote by $S_{c l}^{\tilde{m}}$ the space of classical symbols with complex order $\tilde{m}$ and by $\Psi_{c l}^{\tilde{m}}=\mathrm{Op}\left(S_{c l}^{m}\right)$ the space of classical pseudo-differential operators with complex order $\tilde{m}$.

If $F: \Omega_{1} \rightarrow \Omega_{2}$ is (smooth) diffeomorphism between two bounded open sets $\Omega_{1}, \Omega_{2} \subset \mathbb{R}^{n}$, we keep the same notation for the map $F: C_{c}^{\infty}\left(\Omega_{1}\right) \rightarrow C_{c}^{\infty}\left(\Omega_{1}\right)$ given by $F(f)=f \circ F^{-1}$. For any $A \in \Psi^{m}\left(\Omega_{1}\right)$, the operator

$$
F^{*} A:=F A F^{-1}
$$

is then in $\Psi^{m}\left(\Omega_{2}\right)$. This property allows us to define pseudo-differential operators on manifolds in the following way. Let $M$ be a smooth compact connected manifold of dimension $n$ without boundary. The space $\Psi^{m}(M)$ of pseudo-differential operators of order $m$ on $M$ is the space of operators which are locally transformed by some (and then any) coordinate cover to pseudo-differential operators in $\Psi^{m}\left(\mathbb{R}^{n}\right)$; that is, the operator $A: C^{\infty}(M) \rightarrow \mathcal{D}^{\prime}(M)$ such that there exists a finite open cover $\left(\Omega_{j}\right)_{j}$ of $M$, a subordinate partition of unity $\left(\chi_{j}\right)_{j}$ and diffeomorpshims $F_{j}: \Omega_{j} \rightarrow \mathcal{O}_{j} \subset \mathbb{R}^{n}$ that transform the operators $\chi_{k} A \chi_{j}: C_{c}^{\infty}\left(\Omega_{j}\right) \rightarrow \mathcal{D}^{\prime}\left(\Omega_{k}\right)$ into operators in $\Psi^{m}\left(\mathbb{R}^{n}\right)$.

If $F: \Omega_{1} \rightarrow \Omega_{2}$ is (smooth) diffeomorphism between two bounded open sets $\Omega_{1}, \Omega_{2} \subset \mathbb{R}^{n}$ and if $A \in \Psi_{c l}^{\tilde{m}}\left(\Omega_{1}\right)$, then the operator $F^{*} A$ is then in $\Psi_{c l}^{\tilde{m}}\left(\Omega_{2}\right)$. This property allows us to define pseudo-differential operators on manifolds in the following way. The space $\Psi_{c l}^{\tilde{m}}(M)$ of classical pseudo-differential operators of order $\tilde{m}$ on $M$ is the space of operators which are locally transformed by some (and then any) coordinate cover to classical pseudo-differential operators.

\subsection{Trace expansions}

In this section, we recall trace expansions for pseudo-differential operators.

We start with the trace and kernel expansions due to Seeley, Grubb and Schrohe. Recall that a complex sector is a subset of $\mathbb{C} \backslash\{0\}$ of the form $\Gamma=\Gamma_{I}:=\left\{r e^{i \theta}: r>\right.$ $0, \theta \in I\}$ where $I$ is a subset of $[0,2 \pi]$; it is closed (in $\mathbb{C} \backslash\{0\}$ ) when $I$ is closed. 
Theorem 2.2 [11, Theorem 2.7] Let $M$ be a compact smooth manifold of dimension $n \geq 2$ or let $\Omega$ be a bounded open subset in $\mathbb{R}^{n}$. Let $\mathcal{L} \in \Psi_{\text {cl }}$ be an invertible elliptic operator of order $m_{0} \in \mathbb{N}$. We assume that there exists a complex sector $\Gamma$ such that the homogeneous principal symbol of $\mathcal{L}$ in local coordinates satisfies

$$
\ell_{m_{0}}(x, \xi) \notin-\Gamma^{m_{0}}=\left\{-\mu^{m_{0}}: \mu \in \Gamma\right\} \text { when }|\xi|=1 \text {. }
$$

Let $A \in \Psi_{c l}^{m}$ and let $k \in \mathbb{N}$ such that $-k m_{0}+m<-n$. The kernel $K(x, y, \lambda)$ of $A(\mathcal{L}-\lambda)^{-k}$ is continuous and satisfies on the diagonal

$$
K(x, x, \lambda) \sim \sum_{j=0}^{\infty} c_{j}(x) \lambda^{\frac{n+m-j}{m_{0}}-k}+\sum_{l=0}^{\infty}\left(c_{l}^{\prime}(x) \log \lambda+c_{l}^{\prime \prime}(x)\right) \lambda^{-l-k},
$$

for $\lambda \in-\Gamma^{m_{0}},|\lambda| \rightarrow \infty$, uniformly in closed sub-sectors of $\Gamma$. The coefficients $c_{j}(x)$ and $c_{l}^{\prime}(x)$ are determined from the symbols $a \sim_{h} \sum_{j} a_{m-j}$ and $\ell \sim_{h} \sum_{j} \ell_{m_{0}-j}$ in local coordinates, while the coefficients $c_{l}^{\prime \prime}(x)$ are in general globally determined.

As a consequence, one has for the trace

$$
\operatorname{tr}\left(A(\mathcal{L}-\lambda)^{-k}\right) \sim \sum_{j=0}^{\infty} c_{j} \lambda^{\frac{n+m-j}{m_{0}}-k}+\sum_{l=0}^{\infty}\left(c_{l}^{\prime} \log \lambda+c_{l}^{\prime \prime}\right) \lambda^{-l-k}
$$

where the coefficients are the integrals over $M$ of the traces of the coefficients defined in (2.1).

In the statement above as well as in the rest of the paper, we keep the same notation for an elliptic operator $\mathcal{L}$ and its self-adjoint extension.

Integrating the expansion (2.2) against $\lambda^{z}$ or against $e^{-z \lambda}$ on well chosen $z$-contours yields the following expansions for operators $A$ of any order, see also [29, Section 1]:

Theorem 2.3 Let $\mathcal{L} \in \Psi_{\text {cl }}$ be as in Theorem 2.2.

For any $A \in \Psi_{c l}^{m}$, we have for $t \rightarrow 0$ :

$$
\operatorname{tr}\left(A e^{-t \mathcal{L}}\right) \sim \sum_{j=0}^{\infty} \tilde{c}_{j} t^{\frac{n+m-j}{m_{0}}}+\sum_{l=0}^{\infty}\left(\tilde{c}_{l}^{\prime} \ln t+\tilde{c}_{l}^{\prime \prime}\right) t^{l}
$$

and

$$
\Gamma(t) \operatorname{tr}\left(A \mathcal{L}^{-t}\right) \sim \sum_{j=0}^{\infty} \frac{\tilde{c}_{j}}{s+\frac{n+m-j}{m_{0}}} t^{\frac{n+m-j}{m_{0}}}+\sum_{l=0}^{\infty}\left(\frac{-\tilde{c}_{l}^{\prime}}{(t+l)^{2}}+\frac{\tilde{c}_{l}^{\prime \prime}}{t+l}\right) .
$$

In (2.4), the left had side is meromorphic with poles as indicated by the right hand side. The coefficients $\tilde{c}_{j}, \tilde{c}_{l}^{\prime}$ and $\tilde{c}_{l}^{\prime \prime}$ are multiples of the corresponding $c_{j}, c_{l}^{\prime}$ and $c_{l}^{\prime \prime}$ in (2.2), the actors are universal constants independent of $A$ and $\mathcal{L}$. 
Recently, the real trace expansions were obtained by the author. Their statements require the space $\mathcal{M}^{m}(\mathbb{R})$ of slowly increasing functions, which we now define:

Definition 2.4 For any $m \in \mathbb{R}$, we denote by $\mathcal{M}^{m}(\mathbb{R})$ the Fréchet space of functions $f \in C^{\infty}(\mathbb{R})$ satisfying

$$
\|f\|_{\mathcal{M}^{m}, N}=\sup _{j=0, \ldots, N} \sup _{\lambda \in \mathbb{R}}\langle\lambda\rangle^{j-m}\left|f^{(j)}(\lambda)\right|<\infty, \quad N=0,1, \ldots
$$

The real trace expansions are as follows:

Theorem 2.5 [6] Let $\mathcal{L} \in \Psi_{c l}^{m_{0}}$ be an elliptic self-adjoint operator on a compact manifold $M$ of dimension $n \geq 2$. Its order is $m_{0}>0$. Let $A \in \Psi_{c l}^{m}$ and $\eta \in C^{\infty}(\mathbb{R})$.

(1) If $\eta$ is compactly supported in $(0, \infty)$, then the operator $A \eta(t \mathcal{L})$ is trace-class for all $t \in \mathbb{R}$ and the trace admits the following expansion as $t \rightarrow 0^{+}$,

$$
\operatorname{tr}(A \eta(t \mathcal{L})) \sim c_{m+n} t^{-\frac{m+n}{m_{0}}}+c_{m-n-1} t^{-\frac{m+n-1}{m_{0}}}+\ldots
$$

in the sense that

$$
\operatorname{tr}(A \eta(t \mathcal{L}))-\sum_{j=0}^{N-1} c_{m+n-j} t^{-\frac{m+n-j}{m_{0}}}=O\left(t^{-\frac{m+n-N}{m_{0}}}\right) .
$$

(2) If $m_{0} \geq 1, \Re m>-n$ and $m \notin \mathbb{Z}$ and $\eta \in \mathcal{M}^{m_{\eta}}(\mathbb{R})$ (see Definition 2.4) with $\Re m+m_{\eta} m_{0}<-n$, then the operator $A \eta(t \mathcal{L})$ is traceclass for all $t \in \mathbb{R}$ and the trace admits the following expansion as $t \rightarrow 0^{+}$,

$$
\operatorname{tr}(A \eta(t \mathcal{L}))=\eta(0) c^{\prime}(A)+\sum_{j=0}^{N-1} c_{m+n-j} t^{\frac{-m-n+j}{m_{0}}}+o(1)
$$

where $N \in \mathbb{N}$ is the smallest non-negative integer such that $N \geq m_{0} m_{\eta}+m_{1}+n$. If $\eta$ is equal to a constant near 0 , we need not impose $m_{0} \geq 1$.

(3) In Part (1), the constant $c_{m+n-j}$ are of the form

$$
c_{m+n-j}=c_{m+n-j}^{(\sigma)} c_{m+n-j}^{(\eta)}
$$

where $c_{m+n-j}^{(A)}$ depends only on the poly-homogeneous expansion of the symbol of $A$ in local coordinates and

$$
\tilde{c}_{m+n-j}^{(\eta)}:=\frac{1}{m_{0}} \int_{u=0}^{+\infty} \eta(u) u^{\frac{m-j+n}{m_{0}}} \frac{d u}{u},
$$

and similarly for Part (2).

The relations between the constants in Theorems 2.5 and 2.3 have not been studied. However, it is known that the constant terms are given by the non-commutative residue and the canonical trace, see Propositions 2.7 and 2.9 respectively. 


\subsection{The non-commutative residue}

Here, we recall the definition of the non-commutative residue via local symbols. The original references are $[12,36,37]$. See also $[5,9,29,30]$.

Let $\Omega$ be a bounded open subset in $\mathbb{R}^{n}$ with $n \geq 2$. Let $A \in \Psi_{c l}^{m}(\Omega)$ with symbols $a \sim_{h} \sum_{j \in \mathbb{N}_{0}} a_{m_{j}}$. If $m \in \mathbb{Z}_{n}$, we set

$$
\operatorname{res}_{x}(A):=\int_{\mathbb{S}^{n-1}} a_{-n}(x, \xi) d \varsigma(\xi)
$$

in this paper, $\mathbb{Z}_{n}$ denotes the set

$$
\mathbb{Z}_{n}:=\{-n,-n+1,-n+2, \ldots\}
$$

and $\varsigma$ denotes the surface measure on the Euclidean unit sphere $\mathbb{S}^{n-1} \subset \mathbb{R}^{n}$ which may be obtained as the restriction to $\mathbb{S}^{n-1}$ of the $(n-1)$-form $\varsigma$ defined on $\mathbb{R}^{n}$ by $\sum_{j=1}^{n}(-1)^{j+1} \xi_{j} d \xi_{1} \wedge \ldots \wedge d \xi_{j-1} \wedge d \xi_{j+1} \wedge \cdots \wedge d \xi_{n}$. If $m \in \mathbb{C} \backslash \mathbb{Z}_{n}$, then we set $\operatorname{res}_{x}(A):=0$.

If $F: \Omega_{1} \rightarrow \Omega_{2}$ is (smooth) diffeomorphism between two bounded open sets $\Omega_{1}, \Omega_{2} \subset \mathbb{R}^{n}$ and if $A \in \Psi_{c l}^{m}\left(\Omega_{1}\right)$, then

$$
\left|F^{\prime}(x)\right| \operatorname{res}_{F(x)}\left(F^{*} A\right)=\operatorname{res}_{x}(A) .
$$

Hence res $x$ yields a 1-density on a compact manifold $M$, for which we keep the same notation $\operatorname{res}_{x}(A)$.

Definition 2.6 The function $x \mapsto \operatorname{res}_{x} A$ is the residue density on a bounded open subset $\Omega \subset \mathbb{R}^{n}$ or on an $n$-dimensional compact manifold $M$ with $n \geq 2$. The corresponding integral

$$
\operatorname{res}(A):=\int_{M} \operatorname{res}_{x}(A) \text { or } \operatorname{res}(A):=\int_{\Omega} \operatorname{res}_{x}(A) d x,
$$

is called the non-commutative residue of $A$.

The non-commutative residue is a trace on $\cup_{m \in \mathbb{C}} \Psi_{c l}^{m}$ in the sense that it is a linear functional on $\cup_{m \in \mathbb{C}} \Psi_{c l}^{m}$ which vanishes on commutators. If $M$ is connected, then any other trace on $\cup_{m \in \mathbb{C}} \Psi_{c l}^{m}$ is a multiple of res.

The non-commutative residue also appears in the constant coefficients of the trace expansions recalled in Sect. 2.2:

Proposition 2.7 (1) We continue with the setting and results of Theorem 2.2. The coefficients $c_{0}^{\prime}(x)$ and $c_{0}^{\prime}$ in (2.1) and (2.2) satisfy

$$
c_{0}^{\prime}(x)=\frac{(-1)^{k}}{(2 \pi)^{n} m_{0}} \operatorname{res}_{x}(A) \quad \text { and } \quad c_{0}^{\prime}=\frac{(-1)^{k}}{(2 \pi)^{n} m_{0}} \operatorname{res}(A) .
$$


(2) With the setting and results of Theorem 2.3, we have

$$
\tilde{c}_{0}^{\prime}=\frac{-\operatorname{res}(A)}{(2 \pi)^{n} m_{0}} \text {. }
$$

(3) With the setting and results of Theorem 2.5 , we have when $m \in \mathbb{Z}_{n}$

$$
c_{0}=\frac{1}{m_{0}} \operatorname{res}(A) \int_{0}^{+\infty} \eta(u) \frac{d u}{u} .
$$

Parts (1) and (2) can be found in [29, Section 1], while Part (3) is part of the results in [6].

\subsection{The canonical trace}

In this section, we recall the definition of the canonical trace. References include the original paper [17] by Kontsevich and Vishik, as well as [9,25].

Let $\Omega$ be an open bounded subset of $\mathbb{R}^{n}$ and let $A=\mathrm{Op}(a) \in \Psi_{c l}^{m}(\Omega)$ with complex order $m \notin \mathbb{Z}_{n}$. The symbol of $A$ admits the poly-homogeneous expansion $a \sim_{h} \sum_{j \in \mathbb{N}_{0}} a_{m-j}$. For $x$ fixed, the function $a_{m-j}(x, \cdot)$ is smooth on $\mathbb{R}^{n} \backslash\{0\}$ and ( $m-j$ )-homogeneous with $m-j \notin \mathbb{Z}_{n}$, so [15, Theorem 3.2.3] it extends uniquely into a tempered $(m-j)$-homogeneous distributions on $\mathbb{R}^{n}$ for which we keep the same notation. For each $x \in \Omega$, we define the tempered distributions using the inverse Fourier transform

$$
\kappa_{a, x}=\mathcal{F}^{-1}\{a(x, \cdot)\} \quad \text { and } \quad \kappa_{a_{m-j}, x}=\mathcal{F}^{-1}\left\{a_{m-j}(x, \cdot)\right\}, j=0,1,2, \ldots
$$

The distribution $\kappa_{a_{m-j}, x}$ is $(-n-m+j)$-homogeneous. Then for any positive integer $N$ with $m-N<-n$ and $x \in \Omega$ the distribution $\kappa_{a, x}-\sum_{j=0}^{N} \kappa_{a_{m-j}, x}$ is a continuous function on $\mathbb{R}^{n}$. Furthermore, the function $(x, y) \mapsto \kappa_{a, x}(y)-\sum_{j=0}^{N} \kappa_{a_{m-j}, x}(y)$ is continuous and bounded on $\Omega \times \mathbb{R}^{n}$. Its restriction to $y=0$ is independent of $N>m+n$ and defines the quantity

$$
\operatorname{TR}_{x}(A):=\kappa_{a, x}(0)-\sum_{j=0}^{N} \kappa_{a_{m-j}, x}(0) .
$$

If $F: \Omega_{1} \rightarrow \Omega_{2}$ is diffeomorphism between two bounded open sets $\Omega_{1}, \Omega_{2} \subset \mathbb{R}^{n}$ and if $A \in \Psi_{c l}^{\tilde{m}}\left(\Omega_{1}\right)$, then

$$
\left|F^{\prime}(x)\right| \mathrm{TR}_{F(x)}\left(F^{*} A\right)=\mathrm{TR}_{x}(A) .
$$

Hence, $\mathrm{TR}_{x}$ yields a 1-density on a compact manifold $M$ for which we keep the same notation $\mathrm{TR}_{x}$. 
Definition 2.8 [17] The density $x \mapsto \mathrm{TR}_{x}(A)$ on a compact manifold $M$ or $x \mapsto$ $\mathrm{TR}_{x}(A) d x$ on a bounded open subset $\Omega$ is called the canonical trace density of $A$. The corresponding integral

$$
\operatorname{TR}(A)=\int_{M} \operatorname{TR}_{x}(A) \quad \text { or } \quad \operatorname{TR}(A)=\int_{\Omega} \operatorname{TR}_{x}(A) d x
$$

is called the canonical trace of $A$.

On $\Omega$ or $M$, the map $A \mapsto \mathrm{TR}(A)$ is a linear functional on $\Psi_{c l}^{m}$ for each $m \in \mathbb{C} \backslash \mathbb{Z}_{n}$ and it coincides with the usual $L^{2}$-trace if $\Re m<-n$. It is a trace type functional on $\cup_{m \in \backslash \mathbb{Z}_{n}} \Psi_{c l}^{m}$ in the sense that

$\mathrm{TR}(c A+d B)=c \operatorname{TR}(A)+d \operatorname{TR}(B) \quad$ whenever $\quad c, d \in \mathbb{C}, A, B \in \cup_{m \in \mathbb{C} \backslash \mathbb{Z}_{n}} \Psi_{c l}^{m}$, and

$$
\operatorname{TR}(A B)=\operatorname{TR}(B A) \quad \text { whenever } \quad A B, B A \in \cup_{m \in \mathbb{C} \backslash \mathbb{Z}_{n}} \Psi_{c l}^{m} .
$$

The canonical trace was originally defined in [17], and may be defined on a slightly larger domain [10]. It is related with coefficients in the trace expansions recalled in Sect. 2.2:

Proposition 2.9 (1) We continue with the setting and results of Theorem 2.3. The coefficients $c_{0}^{\prime \prime}$ in (2.2) is equal to the canonical trace $\operatorname{TR}(A)$ of $A$ when $m \notin \mathbb{Z}_{n}$.

(2) With the setting and results of Theorem 2.5 , we have $c^{\prime}(A)=\operatorname{TR}(A)$.

Part (1) can be found in [9, Section 1] while Part (2) is part of the results in [6].

The residue of the canonical trace of a (suitable) holomorphic family of classical pseudo-differential operators is equal to the non-commutative residue. It can also be read off the zeta function of $A$ and in the $\Omega$-setting is related to the finite-part integral of the symbol of $A$ on $\Omega \times \mathbb{R}^{n}$, see also [18] and [6].

\section{The case of the torus}

In this section, we discuss the relations between the pseudo-differential calculi defined on the torus viewed as a compact manifold and defined via the Fourier series.

In this paper, the $n$-dimensional torus is denoted by $\mathbb{T}^{n}$ and is realised as $\mathbb{T}^{n}=$ $\mathbb{R}^{n} / \mathbb{Z}^{n}$.

\subsection{The toroidal pseudo-differential calculus}

In this section we set the notation for and define the toroidal pseudo-differential calculus. References include [1,2,21,22,27,35]. 
A toroidal symbol is a scalar function $\sigma$ defined on $\mathbb{T}^{n} \times \mathbb{Z}^{n}$. The operator $\mathrm{Op}_{\mathbb{T}^{n}}(\sigma)$ associated with the toroidal symbol $\sigma$ is the operator defined via

$$
\mathrm{Op}_{\mathbb{T}^{n}}(\sigma) f(x)=\sum_{\ell \in \mathbb{Z}^{n}} e^{2 i \pi x \cdot \ell} \sigma(x, \ell) \widehat{f}(\ell), \quad x \in \mathbb{T}^{n}
$$

In this formula, $f$ is in the space $L_{\text {finite }}^{2}\left(\mathbb{T}^{n}\right)$ of smooth functions on $\mathbb{T}^{n}$ whose Fourier coefficients

$$
\widehat{f}(\ell)=\mathcal{F}_{\mathbb{T}^{n}} f(\ell)=\int_{\mathbb{T}^{n}} f(x) e^{-2 i \pi x \cdot \ell} d x, \quad \ell \in \mathbb{Z}^{n},
$$

all vanish except for a finite number of them. We will keep the same notation for the operator $\mathrm{Op}_{\mathbb{T}^{n}}(\sigma)$ and its natural extensions between topological vector spaces containing $L_{\text {finite }}^{2}\left(\mathbb{T}^{n}\right)$ as a dense subspace.

For each $j=1, \ldots, n$, we denote by $\Delta_{j}$ the difference operator in the $j$ th direction, that is, the operator acting on toroidal symbols $\sigma$ in the following way:

$$
\Delta_{j} \sigma(x, \ell)=\sigma\left(x, \ell+e_{j}\right)-\sigma(x, \ell), \quad(x, \ell) \in \mathbb{T}^{n} \times \mathbb{Z}^{n},
$$

where $\left(e_{1}, \ldots, e_{n}\right)$ is the canonical basis of $\mathbb{R}^{n}$. The difference operator for the multiindex $\alpha=\left(\alpha_{1}, \ldots, \alpha_{n}\right) \in \mathbb{N}_{0}^{n}$ is denoted by

$$
\Delta^{\alpha}:=\Delta_{1}^{\alpha_{1}} \cdots \Delta_{n}^{\alpha_{n}}
$$

with the convention that $\Delta_{j}^{0}=\mathrm{I}$.

The following statement says that the pseudo-differential operators defined locally on the manifold $M=\mathbb{T}^{n}$ have a global description as toroidal operators of the form $\mathrm{Op}_{\mathbb{T}^{n}}(\sigma)$ :

Theorem 3.1 [27] Let $A \in \Psi^{m}\left(\mathbb{T}^{n}\right)$ for some $m \in \mathbb{R}$. Then there exists a unique toroidal symbol $\sigma_{A}$ such that $A=\mathrm{Op}_{\mathbb{T}^{n}}\left(\sigma_{A}\right)$. The symbol $\sigma_{A}$ is smooth in $x \in \mathbb{T}^{n}$, i.e. $x \mapsto \sigma(x, \ell)$ is smooth for each $\ell \in \mathbb{Z}^{n}$. It satisfies:

$$
\forall \alpha, \beta \in \mathbb{N}_{0}^{n} \quad \exists C>0 \quad \forall(x, \ell) \in \mathbb{T}^{n} \times \mathbb{Z}^{n} \quad\left|\partial_{x}^{\beta} \Delta^{\alpha} \sigma_{A}(x, \ell)\right| \leq C\langle\ell\rangle^{m-|\alpha|} .
$$

Conversely, if a toroidal symbol $\sigma$ is smooth in $x$ and satisfies (3.1), then $\mathrm{Op}_{\mathbb{T}^{n}}(\sigma) \in$ $\Psi^{m}\left(\mathbb{T}^{n}\right)$.

We denote by $S^{m}\left(\mathbb{T}^{n}\right)$ the Fréchet space of toroidal symbols which are smooth in $x$ and satisfy (3.1); we say then that the symbols are of order $m$. There should be no confusion with the notation $S^{m}(\Omega)$ since there $\Omega$ is an open subset of $\mathbb{R}^{n}$. Theorem 3.1 may then be rephrased as

$$
\Psi^{m}\left(\mathbb{T}^{n}\right)=\mathrm{Op}_{\mathbb{T}^{n}}\left(S^{m}\left(\mathbb{T}^{n}\right)\right)
$$


Furthermore, the proof of Theorem 3.1 shows that the map $\sigma \mapsto \mathrm{Op}_{\mathbb{T}^{n}}(\sigma)$ is an isomorphism from $S^{m}\left(\mathbb{T}^{n}\right)$ to $\Psi^{m}\left(\mathbb{T}^{n}\right)$.

We denote by $S^{-\infty}\left(\mathbb{T}^{n}\right)=\cap_{m \in \mathbb{R}} S^{m}\left(\mathbb{T}^{n}\right)$ the set of smoothing toroidal symbols. As a consequence of the results mentioned above, the map $\sigma \mapsto \mathrm{Op}_{\mathbb{T}^{n}}(\sigma)$ is an isomorphism of Fréchet spaces from $S^{-\infty}\left(\mathbb{T}^{n}\right)$ to $\Psi^{-\infty}\left(\mathbb{T}^{n}\right)$.

Naturally, another way of defining globally an operator $A \in \cup_{m \in \mathbb{R}} \Psi^{m}\left(\mathbb{T}^{n}\right)$ is via its integral kernel $K_{A} \in \mathcal{D}^{\prime}\left(\mathbb{T}^{n} \times \mathbb{T}^{n}\right)$ or equivalently via the distribution given by $k_{A, x}(y)=K_{A}(x, x-z)$. Indeed, we have (in the sense of distributions) for any $f \in C^{\infty}\left(\mathbb{T}^{n}\right)$ and $x \in \mathbb{T}^{n}$ :

$$
A f(x)=\int_{\mathbb{T}^{n}} K_{A}(x, y) f(y) d y=\int_{\mathbb{T}^{n}} f(y) \kappa_{A, x}(x-y) d y=f * \kappa_{A, x}(x) .
$$

Recall that the map $x \mapsto \kappa_{A, x} \in \mathcal{D}^{\prime}\left(\mathbb{T}^{n}\right)$ is smooth on $\mathbb{T}^{n}$ and that we have

$$
\forall(x, \ell) \in \mathbb{T}^{n} \times \mathbb{Z}^{n} \quad \widehat{\kappa}_{A, x}(\ell)=\sigma_{A}(x, \ell) \quad \text { where } \sigma_{A}:=\mathrm{Op}_{\mathbb{T}^{n}}^{-1}(A)
$$

Moreover, $\kappa_{A, x}$ is smooth away from the origin for $x$ fixed since $K_{A}$ is smooth away from the diagonal $x=y$. In fact, an operator $A: C^{\infty}\left(\mathbb{T}^{n}\right) \rightarrow \mathcal{D}^{\prime}\left(\mathbb{T}^{n}\right)$ is in $\Psi^{-\infty}\left(\mathbb{T}^{n}\right)$ if and only if $(x, y) \mapsto \kappa_{x}(y)$ is smooth on $\mathbb{T}^{n} \times \mathbb{T}^{n}$.

If $\sigma_{A}$ does not depend on $x$, then $A$ is a Fourier multiplier with symbol $\sigma$ and convolution kernel $\kappa_{A}$. Even when $\sigma_{A}$ depends on $x$, we may abuse the vocabulary and call $\kappa_{A, x}$ the convolution kernel of $A$.

More generally, we have the following property between pseudo-differential and translations:

$$
\tau_{x_{0}}^{-1} \mathrm{Op}_{\mathbb{T}^{n}}(\sigma) \tau_{x_{0}}=\mathrm{Op}_{\mathbb{T}^{n}}\left(\tau_{x_{0}} \sigma\right)
$$

where $\tau_{x_{0}}$ denotes the translation

$$
\tau_{x_{0}} f(x)=f\left(x-x_{0}\right), \quad x_{0} \in \mathbb{T}^{n}, f \in L^{2}\left(\mathbb{T}^{n}\right)
$$

and we abused the notation to have $\tau_{x_{0}} \sigma:(x, \ell) \mapsto \sigma\left(x-x_{0}, \ell\right)$.

As on $\mathbb{R}^{n}$, we say that the toroidal symbol $\sigma \in S^{m}\left(\mathbb{T}^{n}\right)$ admits an expansion and we write $\sigma \sim \sum_{j \in \mathbb{N}_{0}} \sigma_{m-j}$ when $\sigma_{m-j} \in S^{m-j}\left(\mathbb{T}^{n}\right)$ and $\sigma-\sum_{j=0}^{N} \sigma_{m_{j}} \in S^{m-N-1}\left(\mathbb{T}^{n}\right)$.

\subsection{Main results for the classical toroidal pseudo-differential calculus}

As in the Euclidean setting, we say that a toroidal symbol $\sigma$ in $S^{m}\left(\mathbb{T}^{n}\right)$ is classical with complex order $\tilde{m} \in \mathbb{C}$ when it admits a poly-homogeneous expansion $\sigma \sim_{h}$ $\sum_{j \in \mathbb{N}_{0}} \sigma_{\tilde{m}-j}$, that is, when each toroidal symbol $\sigma_{\tilde{m}-j}(x, \ell)$ is in $S^{m-j}\left(\mathbb{T}^{n}\right)$ and is $(\tilde{m}-j)$-homogeneous in $\ell \neq 0$, i.e. $\sigma_{\tilde{m}-j}(x, r \ell)=r^{\tilde{m}-j} \sigma_{\tilde{m}-j}(x, \ell)$ for any $(x, \ell) \in \mathbb{T}^{n} \times\left(\mathbb{Z}^{n} \backslash\{0\}\right), r \in \mathbb{N}$. We denote by $S_{c l}^{\tilde{m}}\left(\mathbb{T}^{n}\right)$ the space of classical symbols of order $\tilde{m} \in \mathbb{C}$. 
The next statement says that the pseudo-differential operators corresponding to classical toroidal (global) symbols are exactly the classical ones on the manifold $M=\mathbb{T}^{n}$ :

Theorem 3.2 For any $m \in \mathbb{C}$, we have

$$
\Psi_{c l}^{m}\left(\mathbb{T}^{n}\right)=\mathrm{Op}_{\mathbb{T}^{n}}\left(S_{c l}^{m}\left(\mathbb{T}^{n}\right)\right)
$$

Theorem 3.2 is a consequence of Proposition 3.4, stated and proved below. Proposition 3.4 is a stronger result, but requires the following property allowing for the identification of a homogeneous toroidal symbol with a homogeneous symbol on $\mathbb{R}^{n}$ :

Proposition 3.3 If $\sigma$ is a toroidal symbol which is $m$-homogeneous in $\ell$ with $m \in \mathbb{C}$, then there exists a unique continuous function in $(x, \xi) \in \mathbb{T}^{n} \times\left(\mathbb{R}^{n} \backslash\{0\}\right)$ which is $m$-homogeneous in $\xi$ and coincides with $\sigma$ on $\mathbb{T}^{n} \times\left(\mathbb{Z}^{n} \backslash\{0\}\right)$. Still denoting by $\sigma$ this unique extension, $\sigma$ is in fact smooth on $\mathbb{T}^{n} \times\left(\mathbb{R}^{n} \backslash\{0\}\right)$.

Proposition 3.3 will be proved in Sect. 3.3. Note that as a consequence of Proposition 3.3, a toroidal symbol which is $m$-homogeneous in $\ell$ is in $S_{c l}^{m}\left(\mathbb{T}^{n}\right), m \in \mathbb{C}$.

Proposition 3.4 whose statement follows may be roughly summarised as saying that the poly-homogeneous expansion of a toroidal symbol is given by the polyhomogeneous expansion in local charts and vice versa:

Proposition 3.4 We fix a finite open covering $\left(\Omega_{k}\right)_{k}$ of $\mathbb{T}^{n}$ and a subordinate partition of unity $\left(\chi_{k}\right)_{k}$ of the form $\chi_{k}(x)=\psi_{k}\left(x-x_{k}\right)$ where the points $x_{k} \in \Omega_{k}$ are distinct and the function $\psi_{k} \in C^{\infty}\left(\mathbb{T}^{n}\right)$ is supported on a small neighbourhood of 0; moreover, $0 \leq \psi_{k} \leq 1$ and $\psi_{k} \equiv 1$ on an even smaller neighbourhood of 0 .

Let $A \in \Psi^{m_{1}}\left(\mathbb{T}^{n}\right)$. Then $\chi_{k_{1}} A \chi_{k_{2}}$ is smoothing for $k_{1} \neq k_{2}$ and if $k_{1}=k_{2}=k$, using the $x_{k}$ translations [see (3.3)] we have

$$
\chi_{k} A \chi_{k}:=\tau_{x_{k}}^{-1} A_{k} \tau_{x_{k}}, \quad \text { where } \quad A_{k}:=\psi_{k} \tau_{x_{k}}^{-1} A \tau_{x_{k}} \psi_{k} .
$$

Denoting by $\sigma=\mathrm{Op}_{\mathbb{T}^{n}}^{-1} A$ the toroidal symbol of $A$, we have $\sigma \in S^{m_{1}}\left(\mathbb{T}^{n}\right)$ by Theorem 3.1.

(1) If $\sigma$ is classical, i.e. $\sigma \in S_{c l}^{m}\left(\mathbb{T}^{n}\right)$ with $\sigma \sim_{h} \sum_{j \in \mathbb{N}_{0}} \sigma_{m-j}$, then the symbol of $A_{k}$ admits the poly-homogeneous expansions $\sum_{j \in \mathbb{N}_{0}} \psi_{k}(x) \sigma_{m-j}\left(x-x_{k}, \xi\right)$ where the functions $\sigma_{m-j}$ have been smoothly extended (see Proposition 3.3).

(2) If $A$ is classical on $\mathbb{T}^{n}$ with complex order $m \in \mathbb{C}$ then $A_{k}$ is classical on $\mathbb{R}^{n}$, its symbol is supported in a small neighbourhood of 0 in $x$ and admits a polyhomogeneous expansion $\sum_{j \in \mathbb{N}_{0}} a_{m-j}^{(k)}$; moreover, $\sigma$ admits a poly-homogeneous expansion which coincides with $\sum_{j \in \mathbb{N}_{0}} a_{m-j}^{(k)}\left(x-x_{k}, \ell\right)$ on $\left\{\chi_{k} \equiv 1\right\}$

In Proposition 3.4 and in its proof (given in Sect. 3.3), we will allow ourselves to make no distinction between functions defined on $\mathbb{T}^{n}$ or $\mathbb{R}^{n}$ when they are supported in a small enough neighbourhood of 0 .

Note that Proposition 3.4 and the local definition of the non-commutative residue (see Sect. 2.3) readily imply: 
Corollary 3.5 If $\sigma \in S_{c l}^{m}\left(\mathbb{T}^{n}\right)$ with $m \in \mathbb{Z}_{n}$, then

$$
\operatorname{res}_{x}(A)=\int_{\mathbb{S}^{n-1}} \sigma_{-n}(x, \xi) d \varsigma(\xi) \text { and } \operatorname{res}(A)=\int_{\mathbb{T}^{n} \times \mathbb{S}^{n-1}} \sigma_{-n}(x, \xi) d x d \varsigma(\xi)
$$

where $\sigma_{-n}$ is the smooth extension of the symbol $\sigma_{-n}$ contained in the expansion $\sigma \sim_{h} \sum_{j \in \mathbb{N}_{0}} \sigma_{m-j}$ as granted by Proposition 3.3.

The expansion results applied to the Laplacian $\Delta$ of $\mathbb{T}^{n}$ easily yields:

Corollary 3.6 Let $\sigma \in S_{c l}^{m}\left(\mathbb{T}^{n}\right)$ with $m \in \mathbb{C}$. We set $A:=\mathrm{Op}_{\mathbb{T}^{n}}(\sigma)$.

(1) Let $\eta \in C_{c}^{\infty}(0, \infty)$. Then we have an expansion as $t \rightarrow 0^{+}$:

$$
\operatorname{tr}(A \eta(t \Delta))=\sum_{\ell \in \mathbb{Z}^{n}} \sigma(x, \ell) \eta\left(t|\ell|^{2}\right) \sim c_{m+n} t^{-\frac{m+n}{m_{0}}}+c_{m-n-1} t^{-\frac{m+n-1}{m_{0}}}+\ldots
$$

The constants $c_{m+n-j}$ are of the form $c_{m+n-j}=c_{m+n-j}^{(\sigma)} c_{m+n-j}^{(\eta)}$ where $c_{m+n-j}^{(\sigma)}$ depends only on the homogeneous expansion of $\sigma$ and

$$
c_{m+n-j}^{(\eta)}:=\frac{1}{2} \int_{u=0}^{+\infty} \eta(u) u^{\frac{m-j+n}{2}} \frac{d u}{u} .
$$

With the same constants $c_{m+n-j}^{(\sigma)}$, if $\Re m>-n$ then we have the expansion as $R \rightarrow+\infty$

$$
\sum_{R \leq|\ell| \leq 2 R} \int_{\mathbb{T}^{n}} \sigma(x, \ell) d x=\sum_{j=0}^{N-1} \frac{2^{m-j+n}-1}{m-j+n} c_{m+n-j}^{(\sigma)} R^{-m+n-j}+o(1),
$$

where $N \in \mathbb{N}$ is the smallest integer such that $\Re m+n-N<0$.

If $m \in \mathbb{Z}_{n}$ then $c_{0}=c_{0}^{(\sigma)} c_{0}^{(\eta)}$ with

$$
c_{0}^{(\sigma)}=\operatorname{res} A, \quad \text { and } \quad c_{0}^{(\eta)}=\frac{1}{2} \int_{0}^{+\infty} \eta(u) \frac{d u}{u}
$$

and we have the expansion as $R \rightarrow+\infty$

$\sum_{R \leq|\ell| \leq 2 R} \int_{\mathbb{T}^{n}} \sigma(x, \ell) d x=\sum_{j=1}^{m+n} \frac{2^{m-j+n}-1}{m-j+n} c_{m+n-j}^{(\sigma)} R^{-m+n-j}+\ln 2 \operatorname{res} A+o(1)$.

(2) Here, $\Re m \geq-n$ with $m \notin \mathbb{Z}$. Let $\eta \in \mathcal{M}^{m_{\eta}}(\mathbb{R})$ (see Definition 2.4) with $\Re m+$ $m_{\eta} m_{0}<-n$. Then the operator $A \eta(t \Delta)$ is traceclass for all $t \in \mathbb{R}$ and the trace admits the following expansion as $t \rightarrow 0^{+}$,

$$
\operatorname{tr}(A \eta(t \Delta))=\eta(0) \operatorname{TR}(A)+\sum_{j=0}^{N-1} c_{m+n-j} t^{\frac{-m-n+j}{2}}+o(1),
$$


where $N \in \mathbb{N}$ is the smallest non-negative integer such that $N \geq 2 m_{\eta}+m_{1}+n$. The constants $c_{m+n-j}^{\prime}$ are of the form $c_{m+n-j}^{\prime}={c^{\prime}}_{m+n-j}^{(\sigma)} c_{m+n-j}^{\prime(\eta)}$ where $c_{m+n-j}^{\prime(\sigma)}$ depends only on the poly-homogeneous expansion of $\sigma$ and

$$
c_{m+n-j}^{\prime(\eta)}:=\frac{1}{2} \int_{u=0}^{+\infty} \eta(u) u^{\frac{m-j+n}{2}} \frac{d u}{u} .
$$

With the same constants ${c^{\prime}}_{m+n-j}^{(\sigma)}$, we have the expansion as $R \rightarrow+\infty$

$$
\sum_{|\ell|<R} \int_{\mathbb{T}^{n}} \sigma(x, \ell) d x=\operatorname{TR}(A)+\sum_{j=0}^{N-1} \frac{c_{m+n-j}^{\prime(\sigma)}}{m-j+n} R^{-m+n-j}+o(1) .
$$

Consequently, the canonical trace of the operator $A=\mathrm{Op}_{\mathbb{T}^{n}}(\sigma)$ is given by the discrete finite part of $\int_{\mathbb{T}^{n}} \sigma(x, \ell) d x$.

Proof of Corollary 3.6 The first results in Part (1) and (2) are directs applications of Theorem 2.5 and Proposition 2.9. For the expansion in $R=t^{-2}$, we apply these results to suitable functions $\chi_{k} \in C_{c}^{\infty}(\mathbb{R})$ approximating the indicator $1_{I}$ of the interval $I=[1,4]$ for Part (1) or of the interval $I=[0,1]$ for Part (2). Suitable approximations are for instance functions satisfying $0 \leq \eta_{k} \leq 1, \eta_{k} \equiv 1$ on $I$ and $\eta_{k} \equiv 0$ outside $I+\left(-\frac{1}{k}, \frac{1}{k}\right)$. As the Laplacian $\Delta$ is invariant under translation, we easily compute for $R>1$

$$
\left.\operatorname{tr}\left(A\left(1_{I}-\eta_{k}\right)\left(R^{-2} \Delta\right)\right)\right)=\sum_{\ell \in \mathbb{Z}^{n}} \int_{\mathbb{T}^{n}} \sigma(x, \ell) d x\left(1_{I}-\eta_{k}\right)\left(R^{-2}|\ell|^{2}\right) .
$$

We also estimate for $I=[1,4]$

$$
\begin{aligned}
\sum_{\ell \in \mathbb{Z}^{n}}\langle\ell\rangle^{\Re m}\left(1_{I}-\eta_{k}\right)\left(R^{-2}|\ell|^{2}\right) & \leq \sum_{\substack{R \sqrt{1-k} \leq|\ell| \leq R \\
\text { or } 2 R \leq|\ell| \leq 2 R \sqrt{1+k}}} \frac{1}{|B(\ell, 1 / 2)|} \int_{B(\ell, 1 / 2)}\langle\xi\rangle^{\Re m} d \xi \\
& \lesssim \int_{\substack{\text { or } 2 R-\frac{1}{2} \leq|\ell| \leq 2 R \sqrt{1+k}+\frac{1}{2} \\
R \sqrt{1-k} \leq|\xi| \leq R+\frac{1}{2}}}\langle\xi\rangle^{\Re m} d \xi \lesssim R^{\Re m+n} \frac{1}{k},
\end{aligned}
$$

and similarly if $I=[0,1]$. Hence, in both cases, we have

$$
\operatorname{tr}\left(A\left(1_{I}-\eta_{k}\right)\left(R^{-2} \Delta\right)\right)=R^{\Re m+n} O\left(\frac{1}{k}\right) .
$$

We easily check the convergences of the coefficients ${c^{\prime}}_{m+n-j}^{\left(\eta_{k}\right)} \longrightarrow_{k \rightarrow+\infty}{c^{\prime}\left(1_{I}\right)}_{m+n-j}$. The results follow by taking $k \rightarrow+\infty$.

A modification of the proofs of Theorem 2.5 Part (2) and Proposition 2.9 Part (2) given in [6] adapted to the case of the torus would also imply that the canonical trace 
density at $x$ of the operator $A=\mathrm{Op}_{\mathbb{T}^{n}}(\sigma)$ is given by the discrete finite part of $\sigma(x, \ell)$. The results regarding the canonical trace on the torus have already been obtained in [20, Section 5]. The particular case of the limit of $\sum_{R \leq|\ell| \leq 2 R} \int_{\mathbb{T}^{n}} \sigma(x, \ell) d x$ in the case $m=-n$ and $R=2^{K}$ have been shown in [26, Theorem 11.15].

\subsection{Proofs of Propositions 3.3 and 3.4}

The main ingredient in the proof of Proposition 3.3 is the following:

Lemma 3.7 Let $\sigma \in S^{0}\left(\mathbb{T}^{n}\right)$ be a toroidal symbol which is independent of $x$ and 0 homogeneous in $\ell \in \mathbb{Z}^{n} \backslash\{0\}$, that is, $\forall \ell \in \mathbb{Z}^{n} \backslash\{0\}$ and $r \in \mathbb{N}, \sigma(r \ell)=\sigma(\ell)$. There exists a unique continuous function $\sigma \in C\left(\mathbb{R}^{n} \backslash\{0\}\right)$ which is 0-homogeneous and coincides with $\sigma$ on $\mathbb{Z}^{n} \backslash\{0\}$. Furthermore $\sigma$ is smooth.

Proof of Lemma 3.7 First, let us show that the estimate

$$
\left|x-x^{\prime}\right| \leq \frac{|x|}{10} \Longrightarrow\left|\sigma\left(x^{\prime}\right)-\sigma(x)\right| \lesssim_{\sigma} \frac{\left|x-x^{\prime}\right|}{|x|}
$$

holds for any $x, x^{\prime} \in \mathbb{Z}^{n} \backslash\{0\}$. We start with the case of $x=\left(x_{1}, \check{x}\right)$ and $x^{\prime}=x+\alpha e_{1}$ with $\alpha \in \mathbb{N}$ and $x_{1} \in \mathbb{N}$ here $\left(e_{1}, \ldots, e_{n}\right)$ denotes the standard orthonormal basis of $\mathbb{R}^{n}$. Since

$$
\sigma(x)-\sigma\left(x+\alpha e_{1}\right)=\sum_{j=0}^{\alpha-1} \Delta_{1} \sigma\left(x+j e_{1}\right),
$$

as $\sigma \in S^{0}\left(\mathbb{T}^{n}\right)$, we have

$$
\begin{aligned}
\left|\sigma(x)-\sigma\left(x+\alpha e_{1}\right)\right| & \lesssim \sigma \sum_{j=0}^{\alpha-1}\left\langle x+j e_{1}\right\rangle^{-1} \lesssim\left|\int_{\left|x_{1}\right|}^{\left|x_{1}+\alpha\right|}(t+|\check{\ell}|)^{-1} d t\right| \\
& \lesssim\left|\ln \frac{\left|x+\alpha e_{1}\right|}{|x|}\right|=|\ln | \frac{x}{|x|}+\frac{\alpha}{|x|} e_{1}|| \lesssim \frac{\alpha}{|x|}
\end{aligned}
$$

when $\alpha \leq|x| / 10$. Hence (3.4) holds when $x^{\prime}=x+\alpha e_{j}$ with $j=1, \alpha \in \mathbb{N}$ and $x_{1} \in \mathbb{N}$. Applying this to $\sigma$ composed with permutations or with $x \mapsto-x$ shows that we have proved (3.4) for any $x, x^{\prime}$ such that the segment $\left[x, x^{\prime}\right]$ is parallel to one of the axes $\mathbb{R} e_{j}$ for some $j$. Writing $x^{\prime}=x+\sum_{j=1}^{n} \alpha_{j} e_{j}$, we then use

$$
\begin{aligned}
\left|\sigma\left(x^{\prime}\right)-\sigma(x)\right| & \leq \sum_{j=1}^{n}\left|\sigma\left(x+\alpha_{1} e_{1}+\cdots+\alpha_{j} e_{j}\right)-\sigma\left(x+\alpha_{1} e_{1}+\cdots+\alpha_{j-1} e_{j-1}\right)\right| \\
& \lesssim \sigma \sum_{j=1}^{n} \frac{\left|\alpha_{j}\right|}{|x|} \lesssim \frac{\left|x-x^{\prime}\right|}{|x|} .
\end{aligned}
$$

This shows (3.4) for any $x, x^{\prime} \in \mathbb{Z}^{n} \backslash\{0\}$. 
Secondly, as $\sigma$ is 0 -homogeneous on $\mathbb{Z}^{n} \backslash\{0\}$, it admits a unique 0-homogeneous extension to $\mathbb{Q}^{n} \backslash\{0\}$, for which we keep the same notation $\sigma$. Note that the homogeneity implies that (3.4) holds for any $x, x^{\prime} \in \mathbb{Q}^{n} \backslash\{0\}$. Let us show that (3.4) allows us for a unique continuous 0 -homogeneous extension to $\mathbb{R}^{n} \backslash\{0\}$. For any $x \in \mathbb{R}^{n} \backslash\{0\}$, consider a sequence $\left(x_{m}\right)_{m \in \mathbb{N}}$ in $\mathbb{Q}^{n}$ converging to $x$. The estimate (3.4) implies that $\left(\sigma\left(x_{m}\right)\right)_{m \in \mathbb{N}}$ is a Cauchy sequence of real numbers, therefore convergent. Furthermore, the limit does not depend on the sequence as the concatenation of two sequences such as $\left(x_{m}\right)_{m \in \mathbb{N}}$ yields a converging sequence. We denote the limit by $\sigma(x)$. This defines an extension of $\sigma$ to $\mathbb{R}^{n} \backslash\{0\}$. Taking limits shows now that (3.4) holds for any $x, x^{\prime} \in \mathbb{R}^{n} \backslash\{0\}$. Consequently, $\sigma$ is continuous on $\mathbb{R}^{n} \backslash\{0\}$, and it is the only such 0-homogeneous extension.

Thirdly, let us show that $\sigma$ is differentiable. We start with the following observation: for every (fixed) $\xi_{0} \in \mathbb{Q}^{n} \backslash\{0\}$, choosing $r_{0} \in \mathbb{N}$ such that $\ell_{0}:=r_{0} \xi_{0} \in \mathbb{Z}^{n}$, we have for any $p, q \in \mathbb{N}$ :

$$
\sigma\left(\xi_{0}+\frac{p}{q} e_{1}\right)-\sigma\left(\xi_{0}\right)-r_{0} p \Delta_{e_{1}} \sigma\left(q \ell_{0}\right)=\sum_{j=1}^{r_{0} p} \Delta_{j e_{1}} \Delta_{e_{1}} \sigma\left(q \ell_{0}\right)
$$

so $\sigma \in S^{0}\left(\mathbb{T}^{n}\right)$ implies

$$
\begin{aligned}
& \left|\Delta_{j e_{1}} \Delta_{e_{1}} \sigma\left(q \ell_{0}\right)\right| \\
& =\left|\sum_{j^{\prime}=1}^{j} \Delta_{e_{1}}^{2} \sigma\left(q \ell_{0}+j^{\prime} e_{1}\right)\right| \lesssim \sigma \sum_{j^{\prime}=1}^{j}\left\langle q \ell_{0}+j^{\prime} e_{1}\right\rangle^{-2} \lesssim \int_{t=1}^{j}\left|q \ell_{0}+t e_{1}\right|^{-2} d t \\
& \quad \lesssim|| q \ell_{0}+\left.j e_{1}\right|^{-1}-\left|q \ell_{0}\right|^{-1} \mid \\
& =\left|q \ell_{0}\right|^{-1}\left(1-\left|\frac{q \ell_{0}}{\left|q \ell_{0}\right|}+\frac{j}{\left|q \ell_{0}\right|} e_{1}\right|^{-1}\right) \lesssim\left|q \ell_{0}\right|^{-2} j,
\end{aligned}
$$

when $1<r_{0} p<q\left|\ell_{0}\right| / 10$, thus

$$
\left|\sigma\left(\xi_{0}+\frac{p}{q} e_{1}\right)-\sigma\left(\xi_{0}\right)-r_{0} p \Delta_{e_{1}} \sigma\left(q \ell_{0}\right)\right| \lesssim \sigma \sum_{j=1}^{r_{0} p}\left|q \ell_{0}\right|^{-2} j \lesssim\left|q \ell_{0}\right|^{-2}\left(r_{0} p\right)^{2}
$$

Adapting the proof above to other cases, it is easy to check that the estimate

$$
\left|\sigma\left(\xi_{0}+h e_{1}\right)-\sigma\left(\xi_{0}\right)-r_{0} p \Delta_{e_{1}} \sigma\left(q \ell_{0}\right)\right| \lesssim\left|\xi_{0}\right|^{2} h^{2}
$$

holds in fact for $h=p / q$ satisfying $p, q \in \mathbb{Z}$, and $|h|<\left|\xi_{0}\right| / 10$. The second observation is that for every $\ell \in \mathbb{Z}^{n} \backslash\{0\}$, the sequence $\left(q \Delta_{e_{1}} \sigma(q \ell)\right)_{q \in \mathbb{N}}$ is bounded because $\sigma \in S^{0}\left(\mathbb{T}^{n}\right)$. Therefore, we can extract a converging subsequence. We can also assume that the subsequence $\left(q_{j}\right)_{j \in \mathbb{N}}$ is the same for all $\ell \in \mathbb{Z}^{n} \backslash\{0\}$. One checks easily that this yields a symbol in $S^{-1}\left(\mathbb{T}^{n}\right)$. As the symbol $|\ell|$ is in $S^{1}\left(\mathbb{T}^{n}\right)$, the symbol 
$\sigma_{1}$ defined via

$$
\sigma_{1}(\ell):=|\ell| \lim _{j \rightarrow \infty} q_{j} \Delta_{e_{1}} \sigma\left(q_{j} \ell\right)
$$

is in $S^{0}\left(\mathbb{T}^{n}\right)$. If $h \in \mathbb{R}$ with $|h|<\left|\xi_{0}\right| / 100$, applying (3.5) with $p_{j}:=\left\lfloor h q_{j}\right\rfloor$ and $q_{j}$, then taking $j \rightarrow+\infty$, we obtain

$$
\left|\sigma\left(\xi_{0}+h e_{1}\right)-\sigma\left(\xi_{0}\right)-\frac{h}{\left|\xi_{0}\right|} \sigma_{1}\left(\ell_{0}\right)\right| \lesssim \sigma\left|\xi_{0}\right|^{2} h^{2} .
$$

This shows that $\partial_{1} \sigma\left(\xi_{0}\right)$ exists when $\xi_{0} \in \mathbb{Q}^{n} \backslash\{0\}$. In particular for the points $\xi_{0}=$ $\ell \in \mathbb{Z}^{n} \backslash\{0\}$, we obtain the convergence

$q \Delta_{e_{1}} \sigma(q \ell)=q\left(\sigma\left(q \ell+e_{1}\right)-\sigma(q \ell)\right)=q\left(\sigma\left(\ell+\frac{1}{q} e_{1}\right)-\sigma(\ell)\right) \longrightarrow_{q \rightarrow+\infty} \partial_{1} \sigma(\ell)$.

This implies that the symbol given by $\partial_{1} \sigma$ is in $S^{-1}\left(\mathbb{T}^{n}\right)$ and is $(-1)$-homogeneous. Therefore, the symbol $\sigma_{1} \in S^{0}\left(\mathbb{T}^{n}\right)$ coincide with $\left\{|\ell| \partial_{1} \sigma(\ell): \ell \in \mathbb{Z}^{n}\right\}$ and is 0 homogeneous. We keep the same notation for its continuous extension $\sigma_{1}$ to $\mathbb{R}^{n} \backslash\{0\}$. The continuity of $\sigma$ and $\sigma_{1}$ together with (3.6) implies that $\partial_{1} \sigma$ exists and is continuous on $\mathbb{R}^{n} \backslash\{0\}$. Naturally the result is true for the other partial derivatives $\partial_{2}, \ldots, \partial_{n}$. This implies that the function $\sigma$ is $C^{1}$ on $\mathbb{R}^{n} \backslash\{0\}$.

Finally, we can also apply this result to $\sigma_{1}$ which is therefore also $C^{1}$. Applying this result recursively and in all the directions shows that $\sigma$ is in fact smooth.

The proof of Proposition 3.3 follows readily:

Proof of Proposition 3.3 The case of $m=0$ is a simple adaptation of the proof of Lemma 3.7 with the addition of the smooth dependence in $x$; it is left to the reader. For any $m \in \mathbb{C}$, it suffices to apply the case $m=0$ to the symbol given by $|\ell|^{-m} \sigma(x, \ell)$ for $\ell \neq 0$.

We can now show Proposition 3.4.

Proof of Proposition 3.4 We observe that the properties of translations [see (3.2)] implies that it suffices to consider the case of $A_{k}$ with $k=0$. The convolution kernel of $A_{0}$ is

$$
\kappa_{x}^{(0)}(y)=\psi_{0}(x) \kappa_{x}(y) \psi_{0}(x-y)
$$

So the symbol of the pseudo-differential operator $A_{0} \in \Psi^{m_{1}}\left(\mathbb{R}^{n}\right)$ is $(x, \xi) \mapsto$

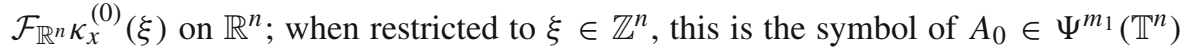
because of the properties of the support of $\kappa^{(0)}$. Hence Part (2) follows. It remains to prove Part (1). By refining the open covering, it suffices to prove the properties in Parts (1) for a neighbourhood of 0 included in $\left\{\psi_{0} \equiv 1\right\}$. Let $V$ be a neighbourhood of 0 included in $\left\{\psi_{0} \equiv 1\right\}$ and let $\chi \in C^{\infty}\left(\mathbb{T}^{n}\right)$ valued in $[0,1]$ with $\chi \equiv 1$ near 0 but 
supported in a neighbourhood of 0 so small that $V+\operatorname{supp} \chi \subset\left\{\psi_{0} \equiv 1\right\}$. Then for $x \in V$, we have

$$
\kappa_{x}^{(0)}(y)=\kappa_{x}(y) \chi(y)+\kappa_{x}(y)(1-\chi)(y) \psi_{0}(x-y) .
$$

The second term is a smoothing kernel, so the symbol of $A_{k}$ is given modulo $S^{-\infty}$ by

$$
\mathcal{F}_{\mathbb{R}^{n}}\left(\kappa_{x} \chi\right)(\xi)=\int_{\mathbb{R}^{n}} \kappa_{x}(y) \chi(y) e^{-2 i \pi y \xi} d y .
$$

The integrand being supported near 0 , we can view this integral as being over $\mathbb{T}^{n}$ and use Parseval formula for Fourier series:

$$
\mathcal{F}_{\mathbb{R}^{n}}\left(\kappa_{x} \chi\right)(\xi)=\sum_{\ell \in \mathbb{Z}^{n}} \sigma(x, \ell) \overline{\mathcal{F}_{\mathbb{T}^{n}}\left\{\chi(y) e^{2 i \pi \cdot \xi}\right\}(\ell)}=\sum_{\ell \in \mathbb{Z}^{n}} \sigma(x, \ell) \overline{\widehat{\chi}(\ell-\xi)}
$$

where $\widehat{\chi}:=\mathcal{F}_{\mathbb{R}^{n}} \chi \in \mathcal{S}\left(\mathbb{R}^{n}\right)$.

Note that

$$
\mathcal{F}_{\mathbb{T}^{n}}\left\{\chi(y) e^{2 i \pi \cdot \xi}\right\}(\ell)=\widehat{\chi}(\ell-\xi)
$$

Furthermore, the Poisson summation formula and the properties of $\chi$ yield

$$
\sum_{\ell \in \mathbb{Z}^{n}} \widehat{\chi}(\ell-\xi)=1 \text { and } \sum_{\ell \in \mathbb{Z}^{n}}(\xi-\ell)^{\alpha} \widehat{\chi}(\ell-\xi)=0
$$

for any $\alpha \in \mathbb{N}_{0}^{n} \backslash\{0\}$ and $\xi \in \mathbb{R}^{n}$.

We assume that $\sigma$ is $m$-homogeneous in $\ell \neq 0$ and we keep the same notation for its smooth homogeneous extension to $\mathbb{T}^{n} \times\left(\mathbb{R}^{n} \backslash\{0\}\right)$ granted by Proposition 3.3. We have for any $N \in \mathbb{N}_{0}$

$$
\mathcal{F}_{\mathbb{R}^{n}}\left\{\chi \kappa_{x}\right\}(\xi)-\sigma(x, \xi)=\sum_{\ell \in \mathbb{Z}^{n}}\left(\sigma(x, \ell)-\sum_{|\alpha| \leq N} \frac{(\xi-\ell)^{\alpha}}{\alpha !} \partial_{\xi}^{\alpha} \sigma(x, \xi)\right) \overline{\widehat{\chi}(\ell-\xi)}
$$

For $r>0$, let $A_{r}:=\left\{\ell \in \mathbb{Z}^{n}: r / 2 \leq|\ell| \leq 2 r\right\}$ denote the set of integers in the annulus with radii $r / 2$ and $2 r$. We decompose the last sum above as $\sum_{\ell \notin A_{|\xi|}}+\sum_{\ell \in A_{|\xi|}}$ and we assume $|\xi| \geq 1$ large. As $\sigma \in C^{\infty}\left(\mathbb{T}^{n} \times\left(\mathbb{R}^{n} \backslash\{0\}\right)\right)$ is $m$-homogeneous, and $\widehat{\chi} \in \mathcal{S}\left(\mathbb{R}^{n}\right)$, for any $N_{1} \in \mathbb{N}$,

$$
\begin{aligned}
\left|\sum_{\ell \notin A_{|\xi|} \mid}\right| & \lesssim \sum_{\ell \notin A_{|\xi|}}\left(|\ell|^{\Re i m}+\langle\xi-\ell\rangle^{N}|\xi|^{\Re i m}\right)\langle\xi-\ell\rangle^{-N_{1}} \\
& \lesssim \sum_{|\ell| \leq|\xi| / 2}|\xi|^{\Re i m+N-N_{1}}+\sum_{|\ell| \geq 2|\xi|}|\ell|^{\Re m+N-N_{1}} \lesssim|\xi|^{\Re m+N-N_{1}+n} .
\end{aligned}
$$


We write the second $\operatorname{sum} \sum_{A_{|\xi|}}$ as $\sum_{A_{|\xi|} \cap B\left(\xi,|\xi|^{1 / 2}\right)}+\sum_{A_{|\xi|} \cap{ }^{c} B\left(\xi,|\xi|^{1 / 2}\right)}$. The Taylor estimates and the homogeneity of $\sigma$ imply

$$
\left|\sum_{A_{|\xi|} \cap B\left(\xi,|\xi|^{1 / 2}\right)}\right| \lesssim \sum_{A_{|\xi|} \cap B\left(\xi,|\xi|^{1 / 2}\right)}|\xi-\ell|^{N+1}|\xi|^{\Re m-(N+1)} \lesssim|\xi|^{\Re m-\frac{N+1}{2}+n}
$$

For the same reasons and because $\widehat{\chi} \in \mathcal{S}\left(\mathbb{R}^{n}\right)$, we have

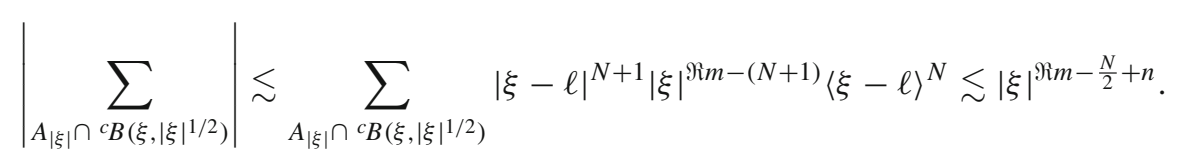

The estimates above imply that for all $N^{\prime} \in \mathbb{N}$ and $\xi \in \mathbb{R}^{n},|\xi| \geq 1$, we have

$$
\left|\mathcal{F}_{\mathbb{R}^{n}}\left\{\chi \kappa_{x}\right\}(\xi)-\sigma(x, \xi)\right| \lesssim \sigma, N^{\prime}|\xi|^{-N^{\prime}} .
$$

We can estimate in the same way $\partial_{x}^{\beta} \partial_{\xi}^{\alpha}\left\{\mathcal{F}_{\mathbb{R}^{n}}\left\{\chi \kappa_{x}\right\}(\xi)-\sigma(x, \xi)\right\}$. This shows that $\mathcal{F}_{\mathbb{R}^{n}}\left\{\chi \kappa_{x}\right\}(\xi)=\chi(x) \sigma$ modulo $S^{-\infty}$ for $x \in V$. By linearity, it is true for any symbol $\sigma \in S_{c l}^{m}\left(\mathbb{T}^{n}\right)$ with $\sigma \sim_{h} \sum_{j \in \mathbb{N}_{0}} \sigma_{m-j}$. This concludes the proof of Proposition 3.4.

\section{Compact Lie groups}

In this section we extend to the non-commutative setting some of the results that were proved in Sect.3. After recalling the setting and its notation in Sect.4.1, we discuss the Hörmander pseudo-differential calculus on a compact Lie group $G$ in Sect.4.2, mainly gathering recent results on the subject. Sections 4.3 and 4.4 are devoted to the descriptions of the principal symbol and of the classical calculus in terms of the global symbol; these are new to our knowledge. The rest of the section is devoted to proofs (Sect.4.5) and to the non-commutative reside and canonical trace on $G$ (Sect. 4.6).

\subsection{Notations and conventions}

In Sect. 4, $G$ always denotes a connected compact Lie group and $n>1$ its dimension. In this section we set the notation and convention regarding the group $G$. References include [13,14,16], see also [7,8].

\subsubsection{Representations}

In this paper, a representation of $G$ is any continuous (hence smooth) group homomorphism $\pi$ from $G$ to the set of automorphisms of a finite dimensional complex vector space. We will denote this space $\mathcal{H}_{\pi}$ with dimension $d_{\pi}=\operatorname{dim} \mathcal{H}_{\pi}$. We may equip $\mathcal{H}_{\pi}$ with an inner product $(\cdot, \cdot)_{\mathcal{H}_{\pi}}$ for which $\pi$ is unitary. The coefficients of $\pi$ are any function of the form $x \mapsto(\pi(x) u, v)_{\mathcal{H}_{\pi}}$ for $u, v \in \mathcal{H}_{\pi}$; these are smooth 
functions on $G$. If a basis $\left\{e_{1}, \ldots, e_{d_{\pi}}\right\}$ of $\mathcal{H}_{\pi}$ is fixed, then we may identify $\mathcal{H}_{\pi}$ with $\mathbb{C}^{d_{\pi}}$, and the matrix coefficients of $\pi$ are the coefficients $\pi_{i, j}, 1 \leq i, j \leq d_{\pi}$ given by $\pi_{i, j}(x)=\left(\pi(x) e_{i}, e_{j}\right) \mathcal{H}_{\pi}$.

We will often identify a representation of $G$ and its equivalence class.

We denote by $\operatorname{Rep}(G)$ and $\widehat{G}$ the sets of representations of $G$ and of irreducible representations of $G$ respectively, both modulo equivalence.

\subsubsection{Lie algebra and vector fields}

The Lie algebra $\mathfrak{g}$ is the tangent space of $G$ at the neutral element $e_{G}$. We denote by $\exp _{G}: \mathfrak{g} \rightarrow G$ the exponential mapping.

We may identify the Lie algebra $\mathfrak{g}$ with the space of left-invariant vector fields on $G$. If a basis $\left\{X_{1}, \ldots, X_{n}\right\}$ for $\mathfrak{g}$ is fixed, we set for any multi-index $\alpha=\left(\alpha_{1}, \ldots, \alpha_{n}\right) \in$ $\mathbb{N}_{0}^{n}$

$$
X^{\alpha}:=X_{1}^{\alpha_{1}} \ldots X_{n}^{\alpha_{n}}
$$

If $\pi$ is a representation of the group $G$, then

$$
\pi(X)=\left.\frac{\partial}{\partial t} \pi\left(\exp _{G}(t X)\right)\right|_{t=0}, \quad X \in \mathfrak{g},
$$

defines a representation also denoted $\pi$ of $\mathfrak{g}$ and therefore of its universal enveloping Lie algebra.

The Lie algebra $\mathfrak{g}$ is the direct sum of the semi-simple Lie algebra $\mathfrak{g}_{s s}=[\mathfrak{g}, \mathfrak{g}]$ with its centre $\mathfrak{z}$ :

$$
\mathfrak{g}=\mathfrak{g}_{s s} \oplus \mathfrak{z} .
$$

Note that $\mathfrak{z}$ is also the Lie algebra of the centre $Z_{G}$ of the group $G$. The Killing form is a scalar inner product on $\mathfrak{g}_{s s} \subset \mathfrak{g}$ and we extend it into a scalar inner product $(\cdot, \cdot)_{\mathfrak{g}}$ on $\mathfrak{g}$ which is invariant under the adjoint action of $G$.

The Casimir element of the universal enveloping algebra is $X_{1}^{2}+\cdots+X_{n}^{2}$ for one and then any orthonormal basis $X_{1}, \ldots, X_{n}$ of $\mathfrak{g}$. Up to a sign, the corresponding differential operator is the (positive) Laplace-Beltrami operator of the compact Lie group $G$ :

$$
\mathcal{L}_{G}:=-X_{1}^{2}-\cdots-X_{n}^{2}
$$

The Laplace-Beltrami operator $\mathcal{L}_{G}$ is a non-negative essentially self-adjoint operator on $L^{2}(G)$. It is a central operator (i.e. invariant under left and right translations) and, for any irreducible representation $\pi$ of $G, \pi(\mathcal{L})$ is scalar:

$$
\pi\left(\mathcal{L}_{G}(\pi)\right)=\lambda_{\pi} \mathrm{I}_{\mathcal{H}_{\pi}}, \quad \pi \in \widehat{G}
$$




\subsubsection{Group Fourier transform}

If $f \in \mathcal{D}^{\prime}(G)$ is a distribution and $\pi$ is a unitary representation, we can define its group Fourier transform at $\pi \in \widehat{G}$ denoted by

$$
\pi(f) \equiv \widehat{f}(\pi) \equiv \mathcal{F}_{G} f(\pi)
$$

via

$$
\pi(f)=\int_{G} f(x) \pi(x)^{*} d x, \text { i.e. } \quad(\pi(f) u, v)_{\mathcal{H}_{\pi}}=\int_{G} f(x)(u, \pi(x) v)_{\mathcal{H}_{\pi}} d x,
$$

since the coefficient functions are smooth. In this paper, the Haar measure $d x$ on $G$ is normalised to be a probability measure.

We denote by $L_{\text {finite }}^{2}(G)$ the set formed by the finite linear combinations of coefficients of representations $\pi \in \widehat{G}$. Hence $L_{\text {finite }}^{2}(G)$ is a vector subspace of $C^{\infty}(G)$. The Peter-Weyl Theorem states that $L_{\text {finite }}^{2}(G)$ is dense in $L^{2}(G)$ and that for any $\pi, \pi^{\prime} \in \widehat{G}, u, v \in \mathcal{H}_{\pi}$ we have

$$
\mathcal{F}_{G}\left\{(\pi(\cdot) u, v)_{\mathcal{H}_{\pi}}\right\}\left(\pi^{\prime}\right)= \begin{cases}d_{\pi}^{-1}(u, v) \mathcal{H}_{\pi} & \text { if } \pi^{\prime}=\pi, \\ 0 & \text { otherwise }\end{cases}
$$

Consequently, a function $f \in C^{\infty}(G)$ admits the following expansion in Fourier series:

$$
f(x)=\sum_{\pi \in \widehat{G}} d_{\pi} \operatorname{tr}(\pi(x) \widehat{f}(\pi))=\sum_{\pi \in \widehat{G}} d_{\pi} \sum_{1 \leq i, j \leq d_{\pi}} \pi_{i, j}(x)[\widehat{f}(\pi)]_{j, i} .
$$

Furthermore the Peter-Weyl Theorem yields an explicit spectral decomposition for $\mathcal{L}_{G}$.

\subsection{The Hörmander pseudo-differential calculus on $G$}

In this section we discuss the Hörmander pseudo-differential calculus on $G$. It may be viewed as a generalisation of the symbols and operator defined on tori in Sect. 3 .

\subsubsection{The symbols and the natural quantisation}

The natural quantisation and notion of symbols on Lie groups was first mentioned by Michael Taylor [34].

On a (connected) compact Lie group $G$, a symbol is a field $\sigma=\{\sigma(x, \pi) \in$ $\left.\mathscr{L}\left(\mathcal{H}_{\pi}\right),(x, \pi) \in G \times \widehat{G}\right\}$ (for a general definition of fields of operators, see e.g. [4, Part II Ch 2]). Here $\mathscr{L}\left(\mathcal{H}_{\pi}\right)$ denotes the set of endomorphisms on the finite dimensional space $\mathcal{H}_{\pi}$, and more generally the space of continuous linear mapping on a Hilbert space $\mathcal{H}_{\pi}$. 
Its associated operator is the operator $\mathrm{Op}_{G}(\sigma)$ defined on $L_{\text {finite }}^{2}(G)$ via

$$
\mathrm{Op}_{G}(\sigma) \phi(x)=\sum_{\pi \in \widehat{G}} d_{\pi} \operatorname{tr}(\pi(x) \sigma(x, \pi) \widehat{\phi}(\pi)), \quad \phi \in L_{\text {finite }}^{2}(G), x \in G .
$$

The Peter-Weyl theorem implies that for $\sigma(x, \pi)=\mathrm{I}_{\mathcal{H}_{\pi}}$ the corresponding operator is the identity. Furthermore, if $T$ is a linear operator defined on $L_{\text {finite }}^{2}(G)$ (and with image some complex-valued functions of $x \in G$ ), then one recovers the symbol via

$$
\sigma(x, \pi)=\pi(x)^{*}(T \pi)(x), \quad \text { that is, }[\sigma(x, \pi)]_{i, j}=\sum_{k} \overline{\pi_{k i}(x)}\left(T \pi_{k j}\right)(x),
$$

when one has fixed a matrix realisation of $\pi$. This shows that the quantisation $\mathrm{Op}_{G}$ defined above is injective.

\subsubsection{Difference operators}

Here we recall the two notions of difference operators, the RT-ones introduced by Michael Ruzhansky and Ville Turunen [27] and the fundamental ones introduced by the author in [8].

If $q \in C^{\infty}(G)$, then the corresponding RT-difference operator $\Delta_{q}$ is defined as acting on Fourier coefficients i.e. $\mathcal{F}_{G}\left(\mathcal{D}^{\prime}(G)\right)$ via

$$
\Delta_{q} \widehat{f}=\mathcal{F}_{G}\{q f\}, \quad f \in \mathcal{D}^{\prime}(G) .
$$

A collection $\Delta=\left\{\Delta_{1}, \ldots, \Delta_{n_{\Delta}}\right\}=\left\{\Delta_{q_{1}}, \ldots, \Delta_{n_{\Delta}}\right\}$ of RT-difference operators is strongly admissible when

$$
\operatorname{rank}\left(\nabla_{e_{G}} q_{1}, \ldots, \nabla_{e_{G}} q_{n_{\Delta}}\right)=n \text { and }\left\{e_{G}\right\}=\cap_{j=1}^{n_{\Delta}}\left\{x \in G: q_{j}(x)=0\right\}
$$

Such a collection exists, see [8,28]. If $\alpha \in \mathbb{N}_{0}^{n_{\Delta}}$, we write $\Delta^{\alpha}=\Delta_{1}^{\alpha_{1}} \ldots \Delta_{n_{\Delta}}^{\alpha_{n}}$. Note that the RT-difference operators are defined to act not on all the symbols but only on $\mathcal{F}_{G}\left(\mathcal{D}^{\prime}(G)\right)$.

Let us now recall the definition of the fundamental difference operators as introduced by the authors in [8], see also [7]. These two references explain how this approach generalises the difference operators of the toroidal case. A symbol $\sigma=$ $\left\{\sigma(x, \pi) \in \mathscr{L}\left(\mathcal{H}_{\pi}\right):(x, \pi) \in G \times \widehat{G}\right\}$ is a field over the set $G \times \widehat{G}$ and the Hilbert space $\oplus_{\pi \in \widehat{G}} \mathcal{H}_{\pi}$ extends naturally to a field over $G \times \operatorname{Rep}(G)$ via

$$
\sigma\left(x, \pi_{1} \oplus \pi_{2}\right)=\sigma\left(x, \pi_{1}\right) \oplus \sigma\left(x, \pi_{2}\right) \in \mathscr{L}\left(\mathcal{H}_{\pi_{1}} \oplus \mathcal{H}_{\pi_{2}}\right) .
$$

Conversely, fields over $G \times \operatorname{Rep}(G)$ satisfying such relations defines a symbol. The difference operator with respect to $\pi_{0} \in \operatorname{Rep}(G)$ is defined via

$$
\Delta_{\pi_{0}} \sigma(x, \pi):=\sigma\left(x, \pi_{0} \otimes \pi\right)-\sigma\left(x, \mathrm{I}_{\mathcal{H}_{\pi_{0}}} \otimes \pi\right), \quad x \in G, \pi \in \operatorname{Rep}(G) .
$$


This defines a field over the set $G \times \widehat{G}$ and the Hilbert space $\oplus_{\pi \in \widehat{G}} \mathcal{H}_{\pi} \otimes \mathcal{H}_{\pi_{0}}$. We also extend this definition to any field over the set $G \times \widehat{G}$ and the Hilbert space $\oplus_{\pi \in \widehat{G}} \mathcal{H}_{\pi} \otimes$ $\mathcal{H}_{\varphi}$ for any $\varphi \in \operatorname{Rep}(G)$. This allows us to compose difference operators. We fix a finite set $\left\{\varphi_{1}, \ldots, \varphi_{f}\right\} \subset \widehat{G}$ of representations of $G$ such that any representation in $\widehat{G}$ will occur in a tensor products of representations in Fund $(G)$. This exists [7], and we call these representations $\varphi_{1}, \ldots, \varphi_{f}$ and the corresponding difference operators $\Delta_{\varphi_{1}}, \ldots, \Delta_{\varphi_{f}}$ fundamental. If $\alpha \in \mathbb{N}_{0}^{f}$, we write $\Delta^{\alpha}=\Delta_{\varphi_{1}}^{\alpha_{1}} \ldots \Delta_{\varphi_{f}}^{\alpha_{f}}$.

\subsubsection{Pseudo-differential calculus on $G$}

The next statement characterises the global symbol of an operator in the Hörmander pseudo-differential calculus on $G$ :

Theorem 4.1 (1) Let $A \in \Psi^{m}(G)$ for some $m \in \mathbb{R}$. Then there exists a unique symbol $\sigma_{A}$ such that $A=\mathrm{Op}_{G}\left(\sigma_{A}\right)$. The symbol is smooth in $x \in G$ in the sense that for each $\pi \in \widehat{G}$, the map $x \mapsto \sigma(x, \pi)$ is smooth on $G$.

- For any basis $X_{1}, \ldots, X_{n}$ of $\mathfrak{g}$ and for any strongly admissible family of $R T$ difference operators $\Delta$, the symbol $\sigma_{A}$ satisfies

$$
\begin{gathered}
\forall \alpha \in \mathbb{N}_{0}^{n_{\Delta}}, \beta \in \mathbb{N}_{0}^{n} \quad \exists C>0 \quad \forall(x, \pi) \in G \times \widehat{G} \\
\left\|X^{\beta} \Delta^{\alpha} \sigma_{A}(x, \pi)\right\|_{\mathscr{L}\left(\mathcal{H}_{\pi}\right)} \leq C\left\langle\lambda_{\pi}\right\rangle^{\frac{m-|\alpha|}{2}} .
\end{gathered}
$$

- The symbol $\sigma_{A}$ satisfies

$$
\begin{gathered}
\forall \alpha \in \mathbb{N}_{0}^{f}, \beta \in \mathbb{N}_{0}^{n} \quad \exists C>0 \quad \forall(x, \pi) \in G \times \widehat{G} \\
\left\|X^{\beta} \Delta^{\alpha} \sigma_{A}(x, \pi)\right\|_{\mathscr{L}} \leq C\left\langle\lambda_{\pi}\right\rangle^{\frac{m-|\alpha|}{2}} .
\end{gathered}
$$

Here $X_{1}, \ldots, X_{n}$ denotes a basis of $\mathfrak{g}$ and we have fixed fundamental representations $\varphi_{1}, \ldots, \varphi_{f}$.

(2) Conversely, the following holds:

- If a symbol $\sigma$ is smooth in $x$ and satisfies (4.1) for a basis $X_{1}, \ldots, X_{n}$ of $\mathfrak{g}$ and for a strongly admissible family of RT-difference operators $\Delta$, then $\mathrm{Op}_{G}(\sigma) \in \Psi^{m}(G)$ and it satisfies (4.1) for any basis $X_{1}, \ldots, X_{n}$ of $\mathfrak{g}$ and for any strongly admissible family of $R T$-difference operators $\Delta$.

- If a symbol $\sigma$ is smooth in $x$ and satisfies (4.1) for a basis $X_{1}, \ldots, X_{n}$ of $\mathfrak{g}$ and for a set fundamental representations $\left\{\varphi_{1}, \ldots, \varphi_{f}\right\}$ of fundamental representations then $\mathrm{Op}_{G}(\sigma) \in \Psi^{m}(G)$ and it satisfies (4.2) for any basis of $\mathfrak{g}$ and any set of fundamental representations.

Theorem 4.1 is shown in [8]. Part (1) is also stated in [27,28], unfortunately with sketches of proofs relying on properties of the pseudo-differential calculus (e.g. composition or properties equivalent to the statement above) which had not been shown in these works. 
We denote by $S^{m}(G)$ the Fréchet space of symbols satisfying (4.2) or equivalently (4.1), and we say then that the symbols are of order $m$. This extends the notation for the toroidal case $G=\mathbb{T}^{n}$ viewed in Sect. 3.1. As in the case of the torus, Theorem 4.1 may then be rephrased as $\Psi^{m}(G)=\mathrm{Op}_{G}\left(S^{m}(G)\right)$. Furthermore, the proof of Theorem 3.1 in [8] shows that the map $\sigma \mapsto \mathrm{Op}_{G}(\sigma)$ is an isomorphism of Fréchet spaces from $S^{m}(G)$ to $\Psi^{m}(G)$. This is so for any $m \in \mathbb{R}$, and also for $m=-\infty$ having denoted by $S^{-\infty}(G)=\cap_{m \in \mathbb{R}} S^{m}(G)$ the set of smoothing symbols.

As on $\mathbb{R}^{n}$ or $\mathbb{T}^{n}$, we say that the symbol $\sigma \in S^{m}(G)$ admits an expansion and we write $\sigma \sim \sum_{j \in \mathbb{N}_{0}} \sigma_{m-j}$ when $\sigma_{m-j} \in S^{m-j}(G)$ and $\sigma-\sum_{j=0}^{m_{N}} \sigma_{m_{j}} \in S^{m-N+1}(G)$.

\subsubsection{Kernels}

As on the torus, another way of defining globally an operator $A \in \cup_{m \in \mathbb{R}} \Psi^{m}(G)$ is via its integral kernel $K_{A} \in \mathcal{D}^{\prime}(G \times G)$ or equivalently via the distribution given by $k_{A, x}(y)=K_{A}\left(x, x z^{-1}\right)$. Indeed, we have (in the sense of distributions) for any $f \in C^{\infty}(G)$ and $x \in G$ :

$$
A f(x)=\int_{G} K_{A}(x, y) f(y) d y=\int_{G} f(y) \kappa_{A, x}\left(y^{-1} x\right) d y=f * \kappa_{A, x}(x) .
$$

Recall that the map $x \mapsto \kappa_{A, x} \in \mathcal{D}^{\prime}(G)$ is smooth on $G$ and that we have

$$
\forall(x, \pi) \in G \times \widehat{G} \quad \widehat{\kappa}_{A, x}(\ell)=\sigma_{A}(x, \pi) \quad\left(\text { where } \sigma_{A}:=\mathrm{Op}_{G}^{-1}(A)\right)
$$

Moreover, $\kappa_{A, x}$ is smooth away from the origin for $x$ fixed since $K_{A}$ is smooth away from the diagonal $x=y$. In fact, an operator $A: C^{\infty}(G) \rightarrow \mathcal{D}^{\prime}(G)$ is in $\Psi^{-\infty}(G)$ if and only if $(x, y) \mapsto \kappa_{x}(y)$ is smooth on $G \times G$.

If $\sigma_{A}$ does not depend on $x$, then $A$ is a group Fourier multiplier with symbol $\sigma$ and convolution kernel $\kappa_{A}$. Even when $\sigma_{A}$ depends on $x$, we may abuse the vocabulary and call $\kappa_{A, x}$ the convolution kernel of $A$.

Lemma 4.2 Let $A=\mathrm{Op}_{G}\left(\sigma_{A}\right) \in \Psi^{m}(G)$ with $m<-n$. Then $\sum_{\pi \in \widehat{G}} d_{\pi} \mid \operatorname{tr}$ $\int_{G} \sigma(x, \pi) d x \mid$ is finite and $A$ is trace-class with

$$
\operatorname{tr}(A)=\int_{G} K_{A}(x, x) d x=\int_{G} \kappa_{A, x}\left(e_{G}\right) d x=\sum_{\pi \in \widehat{G}} d_{\pi} \operatorname{tr} \int_{G} \sigma(x, \pi) d x
$$

Proof The first equality holds on any compact manifold and any operator of order $m<-n$. As $m<-n, \kappa_{x}$ is continuous on $G$ so $\int_{G} K_{A}(x, x) d x=\int_{G} \kappa_{x}\left(e_{G}\right) d x$.

As $\sigma \in S^{m}(G)$, we have

$$
\left|\operatorname{tr} \int_{G} \sigma(x, \pi) d x\right| \leq \sup _{\left(x^{\prime}, \pi^{\prime}\right) \in G \times \widehat{G}}\left\|\sigma\left(x^{\prime}, \pi^{\prime}\right)\right\|\left(1+\lambda_{\pi^{\prime}}\right)^{-\frac{m}{2}} \operatorname{tr}\left(\left(1+\lambda_{\pi}\right)^{-\frac{m}{2}} \mathrm{I}_{\mathcal{H}_{\pi}}\right) .
$$


Recall that the convolution kernel $\mathcal{B}_{s}$ of the operator $\left(\mathrm{I}+\mathcal{L}_{G}\right)^{-\frac{s}{2}}$ is square integrable for $s>n / 2[8$, Lemma A5] and that

$$
\left\|\mathcal{B}_{S}\right\|_{L^{2}(G)}^{2}=\sum_{\pi \in \widehat{G}} d_{\pi}\left\|\left(1+\lambda_{\pi}\right)^{-\frac{s}{2}} \mathrm{I}_{\mathcal{H}_{\pi}}\right\|_{H S}^{2}=\sum_{\pi \in \widehat{G}} d_{\pi} \operatorname{tr}\left(1+\lambda_{\pi}\right)^{-s} \mathrm{I}_{\mathcal{H}_{\pi}} .
$$

In this paper, $\|\cdot\|_{H S}$ denotes the Hilbert-Schmidt norm of a matrix or an operator on a Hilbert space. Therefore, as $m<-n$, we have

$$
\begin{aligned}
& \sum_{\pi \in \widehat{G}} d_{\pi}\left|\operatorname{tr} \int_{G} \sigma(x, \pi) d x\right| \\
& \quad \leq \sup _{\left(x^{\prime}, \pi^{\prime}\right) \in G \times \widehat{G}}\left\|\sigma\left(x^{\prime}, \pi^{\prime}\right)\right\|\left(1+\lambda_{\pi^{\prime}}\right)^{-\frac{m}{2}} \sum_{\pi \in \widehat{G}} d_{\pi} \operatorname{tr}\left(\left(1+\lambda_{\pi}\right)^{-\frac{m}{2}} \mathrm{I}_{\mathcal{H}_{\pi}}\right) \\
& =\sup _{\left(x^{\prime}, \pi^{\prime}\right) \in G \times \widehat{G}}\left\|\sigma\left(x^{\prime}, \pi^{\prime}\right)\right\|\left(1+\lambda_{\pi^{\prime}}\right)^{-\frac{m}{2}}\left\|\mathcal{B}_{-m / 2}\right\|^{2},
\end{aligned}
$$

and the sum on the right-hand side is finite. Moreover, since $\int_{G} \sigma(x, \pi) d x=$ $\mathcal{F}_{G}\left\{\int_{G} \kappa_{x} d x\right\}(\pi)$, the Fourier inversion formula yields:

$$
\int_{G} \kappa_{x}\left(e_{G}\right) d x=\sum_{\pi \in \widehat{G}} d_{\pi} \operatorname{tr} \int_{G} \sigma(x, \pi) d x .
$$

\subsubsection{Invariance of the calculi under translations}

For any operator $A=\mathrm{Op}_{G}\left(\sigma_{A}\right)$ and $x_{0} \in G$, we denote by $x_{0} A$ and $A_{x_{0}}$ the left and right translated of $A$, that is, the operators given by

$$
x_{0} A(f)(x)=A\left(f\left(x_{0}^{-1} \cdot\right)\right)\left(x_{0} x\right) \text { and } A_{x_{0}}(f)(x)=A\left(f\left(\cdot x_{0}^{-1}\right)\right)\left(x x_{0}\right) .
$$

We check easily that the symbols of ${ }_{x_{0}} A$ and $A_{x_{0}}$ are given by respectively:

$$
\sigma_{x_{0} A}(x, \pi)=\sigma_{A}\left(x_{0} x, \pi\right) \text { and } \sigma_{A_{x_{0}}}(x, \pi)=\pi\left(x_{0}\right) \sigma_{A}\left(x x_{0}, \pi\right) \pi\left(x_{0}\right)^{-1} .
$$

It follows readily from the definition of the symbol classes [see the conditions in (4.2)] that for any $x_{0} \in G$ we have

$$
\sigma_{A} \in S^{m}(G) \Longrightarrow \sigma_{x_{0}} A \text { and } \sigma_{A_{x_{0}}} \text { are in } S^{m}(G)
$$

Furthermore, the maps $\sigma_{A} \mapsto \sigma_{x_{0}} A$ and $\sigma_{A} \mapsto \sigma_{A_{x_{0}}}$ are continuous isomorphisms of the Fréchet space $S^{m}(G)$. Consequently, $\Psi^{m}(G)$ is invariant under left or right translations in the sense that for any $x_{0} \in G$ we have:

$$
A \in \Psi^{m}(G) \Longrightarrow x_{0} A \text { and } A_{x_{0}} \text { are in } \Psi^{m}(G) .
$$


Furthermore, we can integrate with respect to $x_{0} \in G$ :

$$
A \in \Psi^{m}(G) \Longrightarrow \int_{G} x_{0} A d x_{0} \text { and } \int_{G} A_{x_{0}} d x_{0} \text { are in } \Psi^{m}(G) \text {. }
$$

Let us prove a similar result for the classical calculus:

Proposition 4.3 For any $A \in \Psi_{c l}^{m}(G)$ and $x_{0} \in G$, the operators $x_{0} A, A_{x_{0}}, \int_{G} x_{0} A d x_{0}$ and $\int_{G} A_{x_{0}} d x_{0}$ are in $\Psi_{c l}^{m}(G)$.

Proof We can construct a finite open cover of $G$ of the form $\left(\Omega z_{j}\right)_{j}$ where $\Omega$ is a small neighbourhood of $e_{G}$ and $\left(z_{j}\right)_{j}$ distinct point on $G$. We can also find a subordinate partition of unity of the form $\chi_{j}=\psi_{j}\left(\cdot z_{j}\right)$, and $\psi_{j}$ valued in $[0,1]$, identically 1 near $e_{G}$ and with a small support about $e_{G}$. The exponential mapping $\exp _{G}$ is a smooth diffeomorphism from a neighbourhood of 0 onto a neighbourhood of supp $\psi_{j}$. For any $A: C^{\infty}(G) \rightarrow \mathcal{D}^{\prime}(G)$, we define $A_{j, k}: C_{c}^{\infty}(\Omega) \rightarrow \mathcal{D}^{\prime}(\Omega)$ via

$$
A_{j, k}(f)(x)=\left(\chi_{k} A \chi_{j}\left(f\left(\cdot x_{j}\right)\right)\right)\left(x x_{k}^{-1}\right) .
$$

Then $A: C^{\infty}(G) \rightarrow \mathcal{D}^{\prime}(G)$ is in $\Psi^{m}(G)$ if and only if all the operators $\phi \mapsto$ $\left(A_{j, k}\left(\phi \circ \exp _{G}^{-1}\right)\right) \circ \exp _{G}$ are in $\Psi^{m}(\mathcal{O})$. And $A \in \Psi^{m}(G)$ is classical if and only if all the operators $\phi \mapsto\left(A_{j, k}\left(\phi \circ \exp _{G}^{-1}\right)\right) \circ \exp _{G}$ are in $\Psi_{c l}^{m}(\mathcal{O})$.

We observe that $\left(x_{0} A\right)_{j, k}$ coincides with $A_{j, k}^{\prime}$ constructed in a similar fashion, but with the $z_{j}$ being replaced by $x_{0}^{-1} z_{j}$. The membership of $x_{0} A$ follows readily. Furthermore, all the operators $\left(x_{0} A\right)_{j, k}$ have an integral kernel which depends smoothly on $x_{0}$, i.e. $G \ni x_{0} \mapsto K_{\left(x_{0} A\right)_{j, k}} \in \mathcal{D}^{\prime}(G \times G)$ is continuous; therefore the Euclidean symbols of $\phi \mapsto\left(\left(x_{0} A\right)_{j, k}\left(\phi \circ \exp _{G}^{-1}\right)\right) \circ \exp _{G}$ depend smoothly on $x_{0}$ as well. Hence, one checks easily that the integration over $G$ also produces a classical symbols. This implies the membership of $\int_{G} x_{0} A d x_{0}$ in $\Psi_{c l}^{m}(G)$.

Similarly, we obtain the $\Psi_{c l}^{m}(G)$-memberships of $A_{x_{0}}$ by considering left translated of $\Omega$ and of $\int_{G} A_{x_{0}} d x_{0}$ as above.

The proof of Proposition 4.3 yields readily:

Corollary 4.4 Let $A \in \Psi_{c l}^{m}(G)$ and let $x_{0} \in G$.

(1) Denoting by $a_{m}$ the principal symbol of $A$, the principal symbols of $x_{0} A$ and $A_{x_{0}}$ at $x$ are given by $a_{m}\left(x_{0} x, L_{x_{0}}^{*} \cdot\right)$ and $a_{m}\left(x x_{0}, R_{x_{0}}^{*} \cdot\right)$ where $L_{x_{0}}^{*}$ and $R_{x_{0}}^{*}$ are the pullbacks of the left and right $x_{0}$-translation mappings.

(2) Translating the operator yields translation of the residue density

$$
\operatorname{res}_{x_{1}}\left(x_{0} A\right)=\operatorname{res}_{x_{0} x_{1}}(A) \quad \text { and } \quad \operatorname{res}_{x_{1}}\left(A_{x_{0}}\right)=\operatorname{res}_{x_{1} x_{0}}(A) \text {, }
$$

and of the canonical trace density:

$$
\mathrm{TR}_{x_{1}}\left(x_{0} A\right)=\mathrm{TR}_{x_{0} x_{1}}(A) \quad \text { and } \quad \operatorname{TR}_{x_{1}}\left(A_{x_{0}}\right)=\operatorname{TR}_{x_{1} x_{0}}(A)
$$


Consequently, the operators $A, x_{0} A, A_{x_{0}}, \int_{G} x_{0} A d x_{0}$ and $\int_{G} A_{x_{0}} d x_{0}$ have the same non-commutative residue and the same canonical trace. If $m<-n$, they have the same trace.

We will not use Part (2) of this corollary in this paper.

\subsection{Principal symbols}

Our definition of homogeneous symbol is motivated by the following important property for which we need the following conventions. Recall that for a non-trivial (unitary) representation $\pi$ when a maximal torus $T$ is fixed, the representation space $\mathcal{H}_{\pi}$ decomposes orthogonally into $\pi(T)$-eigenspaces. The corresponding non-zero eigenvalues form the set of weights. We will also use the notion of analytical integral weight. Some well-known facts will also be recalled and used below. References for this classical material include [16] (especially Chapters IV and V) and [13] (especially Section 12).

Lemma 4.5 Let $A=\mathrm{Op}_{G}\left(\sigma_{A}\right) \in \Psi_{c l}^{0}(G)$. Let $\chi_{1}, \chi_{2} \in C^{\infty}(G)$ with small supports near $e_{G}$ and identically equal to 1 on a small neighbourhood of $e_{G}$. Let $b \in S^{0}\left(\mathbb{R}^{n}\right)$ be the principal symbol of the operator $\exp ^{*} \chi_{1} A \chi_{2}: f \mapsto\left(\chi_{1} A\left(\left(\chi_{2} f\right) \circ \exp _{G}\right)\right) \circ \exp _{G}^{-1}$. Let $w \in \mathfrak{g}^{*}$. If there exists a maximal torus $T$ of $G$ and a non-trivial irreducible representation $\pi$ of $G$ such that $w$ is an analytical integral weight for $\pi$ and $T$ then

$$
b(0, w)=\lim _{k \rightarrow+\infty}\left(\sigma_{A}\left(e_{G}, \pi^{\otimes k}\right) v^{\otimes k}, v^{\otimes k}\right)_{\mathcal{H}_{\pi}^{\otimes k}},
$$

where $v$ is a unit $w$-weight vector.

As the eigenspace of an analytical integral weight is one-dimensional, the limit relation in Lemma 4.5 does not depend on the choice of a unit highest weight vector.

Proof of Lemma 4.5 If $f$ is a smooth function supported in a neighbourhood small enough of $e_{G}$ and if $X$ is in a neighbourhood small enough of $e_{G}$, we have:

$$
\begin{aligned}
A\left(f \circ \exp _{G}^{-1}\right)\left(\exp _{G}(X)\right) & =\int_{G} f \circ \exp _{G}^{-1}(y) \kappa_{A, \exp _{G}(X)}\left(y^{-1} \exp _{G}(X)\right) d y \\
& =\int_{\mathfrak{g}} f(Y) \kappa_{A, \exp _{G}(X)}\left(\exp _{G}(-Y) \exp _{G}(X)\right)\left|\operatorname{jac}_{Y} \exp \right| d Y
\end{aligned}
$$

after the change of variable $y=\exp _{G}(Y)$. Hence the symbol $a$ of the operator $\exp ^{*} \chi_{1} A \chi_{2}$ is, up to a smoothing symbol, given by

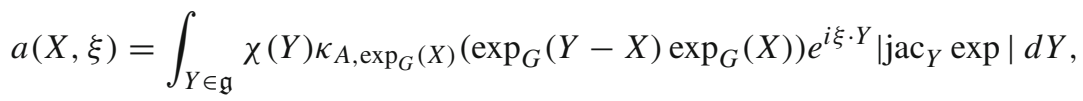

where $\chi \in C_{c}^{\infty}(\mathbb{R})$ is valued in $[0,1]$ and supported in a small neighbourhood of 0 with $\chi \equiv 1$ near 0 . Consequently, its principal symbol at $X=0$ is

$$
b(0, \xi)=\lim _{r \rightarrow+\infty} \int_{Y \in \mathfrak{g}} \chi(Y) \kappa_{A, e_{G}}\left(\exp _{G}(Y)\right) e^{i r \xi \cdot Y}\left|\mathrm{jac}_{Y} \exp \right| d Y .
$$


Let $w \in \mathfrak{g}^{*}$ such that there exists a maximal torus $T$ of $G$ and a non-trivial irreducible representation $\pi$ of $G$ such that $w$ is an analytical integral weight for $\pi$ and $T$. We fix an ordering on $\mathfrak{g}^{*}$ so that $w$ is dominant and has become the highest weight of $\pi$. Let $v$ be a unit highest weight vector. For every $k \in \mathbb{N}$, the highest weight theory yields:

$$
\forall y \in G \quad\left(\pi_{k w}(y) v_{k w}, v_{k w}\right) \mathcal{H}_{\pi_{k w}}=\left(\pi^{\otimes k}(y) v^{\otimes k}, v^{\otimes k}\right)_{\mathcal{H}_{\pi}^{\otimes k}}
$$

where $\pi_{k w}$ is the representation with highest weight $k w$ and $v_{k w}$ a unit highest weight vector. Therefore, we have for any $x \in G$

$$
\begin{aligned}
\left(\sigma_{A}\left(x, \pi^{\otimes k}\right) v^{\otimes k}, v^{\otimes k}\right) \mathcal{H}_{\pi \otimes k} & =\left(\sigma_{A}\left(x, \pi_{k w}\right) v_{k w}, v_{k w}\right) \mathcal{H}_{\pi_{k w}} \\
& =\left(\widehat{\kappa}_{A, x}\left(\pi_{k w}\right) v_{k w}, v_{k w}\right) \mathcal{H}_{\pi_{k w}} .
\end{aligned}
$$

Setting $\chi_{G}=\chi \circ \exp$, we have $\widehat{\kappa}_{A, e_{G}}=\mathcal{F}_{G}\left(\chi_{G} \kappa_{A, e_{G}}\right)+\mathcal{F}_{G}\left(\left(1-\chi_{G}\right) \kappa_{A, e_{G}}\right)$, and the second term is smoothing, so

$\left|\left(\mathcal{F}_{G}\left(\left(1-\chi_{G}\right) \kappa_{A, e_{G}}\right)\left(\pi_{k w}\right) v_{k w}, v_{k w}\right) \mathcal{H}_{\pi_{k w}}\right| \leq\left\|\mathcal{F}_{G}\left(\left(1-\chi_{G}\right) \kappa_{A, e_{G}}\right)\left(\pi_{k w}\right)\right\| \mathscr{L}_{\left(\mathcal{H}_{\pi_{k w}}\right)}$

for any $N \in \mathbb{N}$. By [7, Lemma 3.8], $\left\langle\lambda_{\pi_{k w}}\right\rangle \asymp\langle k w\rangle^{2} \asymp k^{2}$ for $w$ fixed. For the first term, we use

$$
\left(\pi_{k w}\left(e^{Y}\right) v_{k w}, v_{k w}\right) \mathcal{H}_{\pi_{k w}}=e^{i k w(Y)}
$$

for $Y$ in a small neighbourhood of 0 to obtain:

$$
\left(\mathcal{F}_{G}\left(\chi_{G} \kappa_{A, e_{G}}\right)\left(\pi_{k w}\right) v_{k w}, v_{k w}\right) \mathcal{H}_{\pi_{k w}}=\int_{\mathfrak{g}} \chi(Y) \kappa_{A, e_{G}}\left(\exp _{G}(Y)\right) e^{k i w(Y)}\left|\operatorname{jac}_{Y} \exp \right| d Y
$$

This implies easily the limit relation in the statement.

Having chosen a maximal torus $T$ in $G$, the set of analytical integral functionals

$$
\left\{w \text { analytical integral weight for } \pi \text { and } T: \pi \in \widehat{G} \backslash\left\{1_{\widehat{G}}\right\}\right\} \text {, }
$$

form a lattice of the dual $\mathfrak{t}^{*}$ of the Lie algebra $\mathfrak{t}$ of $T$ (see also [7, Section 2.4] for a discussion with this viewpoint). It is easily seen that given any real finite-dimension vector space $V$, a continuous homogeneous function on $V \backslash\{0\}$ is characterised by its restriction to any lattice of $V$. Hence, Lemma 4.5 implies that, keeping its notation, $b(0, \cdot)$ is determined completely on $\mathfrak{t}^{*} \backslash\{0\}$. Now the unions of all the possible maximal tori and of the duals of their Lie algebras are $G$ and $\mathfrak{g}^{*}$ respectively. Therefore, $b$ is determined completely on $\{0\} \times \mathfrak{g}^{*} \backslash\{0\}$. The properties of translations in Corollary 4.4 Part (1) then yield:

Theorem 4.6 Let $A=\mathrm{Op}_{G}\left(\sigma_{A}\right) \in \Psi_{c l}^{0}(G)$. Using the homogeneity and the pullback of the left translations, its principal symbol is identified with a smooth function $a_{0}$ : 
$G \times\left(\mathfrak{g}^{*} \backslash\{0\}\right) \rightarrow \mathbb{C}$ which is homogeneous of degree 0 in the variable $\xi \in \mathfrak{g}^{*} \backslash\{0\}$. Then we have for $x \in G$ and any non-trivial $\pi \in \widehat{G}$,

$$
a_{0}(x, w)=\lim _{k \rightarrow+\infty}\left(\sigma_{A}\left(x, \pi^{\otimes k}\right) v^{\otimes k}, v^{\otimes k}\right)_{\mathcal{H}_{\pi}^{\otimes k}},
$$

where $w$ and $v \in \mathcal{H}_{\pi}$ are the highest weight and a corresponding unit vector for a given maximal torus $T$ and ordering on $\mathfrak{g}^{*}$. This characterises $a_{0}$.

This theorem shows how to obtain the principal symbol $a_{0}$ from the global symbol $\sigma_{A}$. The matrix symbol contains all the information about the operator $A$ whereas the principal symbol may be viewed as the operator $A$ modulo $\cup_{m<0} \Psi^{m}(G)$.

In the case of the torus $G=\mathbb{T}^{n}$, every representation $\pi \in \widehat{G}$ is of dimension 1 and identified with a character $x \mapsto e^{2 i \pi x \cdot \ell}$; furthermore, $\pi^{\otimes k}$ corresponds then to the character $x \mapsto e^{2 i \pi x \cdot(k \ell)}$. With Theorem 4.6, we recover

$$
a_{0}(x, \ell)=\lim _{k \rightarrow+\infty} \sigma_{A}(x, k \ell)
$$

which is a simple consequence of Proposition 3.4.

\subsection{Homogeneous symbols and classical symbols on $G$}

We are led to define the following notion of homogeneity.

Definition 4.7 Let $\sigma$ be a symbol. It is said to be 0 -homogeneous when the following condition is satisfied for every $x \in G$ and every $\pi \in \widehat{G} \backslash\{1\}$ of $G$. We fix a maximal torus $T$ and a realisation of $\pi$ as a unitary irreducible representation of $G$. We consider an orthonormal basis of $\pi(T)$-eigenvectors for the representation space. The only possibly non-zero coefficients of $\sigma(x, \pi)$ are the diagonal ones corresponding to analytical integral weights, i.e. $\left(\sigma(x, \pi) v_{1}, v_{2}\right)=0$ if $v_{1}, v_{2}$ are $\pi(T)$-eigenvectors for weights which are not both analytical integral, or if $v_{1}, v_{2}$ are $\pi(T)$-eigenvectors for distinct analytical integral weights. Furthermore, if $v$ is a unit eigenvector for an analytical integral weight for $\pi$ and $T$, then we have for every $k \in \mathbb{N}$

$$
(\sigma(x, \pi) v, v)_{\mathcal{H}_{\pi}}=\left(\sigma\left(x, \pi^{\otimes k}\right) v^{\otimes k}, v^{\otimes k}\right) \mathcal{H}_{\pi} \otimes k
$$

In the last relation, $\pi^{\otimes k}$ denotes the $k$-tensor product of the representation $\pi$. As $v$ is a unit eigenvector for an analytical integral weight $w$ for $\pi$ and $T, v^{\otimes k}$ is a unit eigenvector for the analytical integral weight $k w$ for $\pi^{\otimes k}$ and $T$. Furthermore, this last relation does not depend on the choice of the unit eigenvector $v$ since the eigenspace of an analytical integral weight is one-dimensional. In fact, it implies

$$
(\sigma(x, \pi) v, v)_{\mathcal{H}_{\pi}}=\left(\sigma\left(x, \pi_{k w}\right) v_{k w}, v_{k w}\right)_{\mathcal{H}_{\pi_{w k}}}
$$

where $\pi_{k w}$ is the representation with highest weight $k w$ and $v_{k w}$ a unit highest weight vector, see (4.3). 
Definition 4.7 gives a condition on the symbol $\sigma$ as a field over $G \times \widehat{G}$. Indeed, if $\pi_{1}$ is equivalent to the representation $\pi$ in the definition, then $\pi_{1}$ and $\pi$ are isomorphic and therefore have the same matrix representation in an orthonormal basis of weight vectors. Furthermore, the condition in Definition 4.7 does not depend on the choice of a maximal torus $T$. Indeed, if $T^{\prime}$ is another maximal torus $T$ then $T^{\prime}$ and $T$ are conjugate by an element $g$ of $G$; the co-adjoint action at $g$ is an isomorphism for the two sets of weights and the unitary map $\pi(g)$ intertwines the matrix representation in the basis of eigenvectors for $\pi(T)$ and $\pi\left(T^{\prime}\right)$.

We can now define our notion of classical poly-homogeneous expansion for our global symbol in $G$ :

Definition 4.8 A symbol $\sigma$ is said to be $m$-homogeneous with $m \in \mathbb{C}$ when $\lambda_{\pi}^{m / 2} \sigma(x, \pi)$ yields a 0 -homogeneous symbol.

A symbol is classical of complex order $m \in \mathbb{C}$ when $\sigma$ is in $S^{\Re i m}(G)$ and admits the expansion $\sigma \sim_{h} \sum_{j \in \mathbb{N}_{0}} \sigma_{m-j}$ where each $\sigma_{m-j}$ is in $S^{\Re m-j}$ and is $(m-j)$ homogeneous.

In the case of the group being a torus $G=\mathbb{T}^{n}$, this definition is consistent with the ones given in Sect.3.2. Therefore, we can generalise the notation of that section and denote by $S_{c l}^{m}(G)$ the space of classical symbol of order $m \in \mathbb{C}$. We obtain as in the case of the torus:

Theorem 4.9 For any $m \in \mathbb{C}$, we have

$$
\Psi_{c l}^{m}(G)=\mathrm{Op}_{G}\left(S_{c l}^{m}(G)\right) .
$$

The next section is devoted to the proof of Theorem 4.9.

\subsection{Proof of Theorem 4.9}

Let us first show the inclusion $\mathrm{Op}_{G}\left(S_{c l}^{m}(G)\right) \subset \Psi_{c l}^{m}(G)$. By linearity of $\mathrm{Op}_{G}$ and the result on $\cup_{m_{1} \in \mathbb{R}} \Psi^{m_{1}}(G)$, it suffices to show:

Lemma 4.10 If $\sigma \in S^{\Re i m}$ is m-homogeneous, then $\mathrm{Op}_{G}(\sigma) \in \Psi_{c l}^{m}(G)$.

Proof of Lemma 4.10 We already know $A:=\mathrm{Op}_{G}(\sigma) \in \Psi^{\Re m}(G)$. If $\chi \in C_{c}^{\infty}(\mathbb{R})$ has a small enough support near 0 with $\chi(0)=1$, then the operator spectrally defined via $\chi\left(\mathcal{L}_{G}\right)$ is the projection onto the constant function and $\mathcal{L}_{G}+\chi\left(\mathcal{L}_{G}\right)$ is injective. By [8, Proposition 3.14], the operator $\chi\left(\mathcal{L}_{G}\right)$ is smoothing and $\left(\mathcal{L}_{G}+\chi\left(\mathcal{L}_{G}\right)\right)^{-m_{1} / 2} \in$ $\Psi^{\Re m_{1}}(G)$ for any $m_{1} \in \mathbb{C}$. Furthermore, the properties of a Laplace operator on a compact manifold [31] imply $\left(\mathcal{L}_{G}+\chi\left(\mathcal{L}_{G}\right)\right)^{-m_{1} / 2} \in \Psi_{c l}^{m_{1}}(G)$. The properties of $\mathrm{Op}_{G}$ yield $A_{1}:=A\left(\mathcal{L}_{G}+\chi\left(\mathcal{L}_{G}\right)\right)^{-m / 2}:=\mathrm{Op}_{G}\left(\sigma_{1}\right)$ where $\sigma_{1}$ is the symbol given by

$$
\sigma_{1}\left(x, 1_{\widehat{G}}\right):=\sigma\left(x, 1_{\widehat{G}}\right) \quad \text { and } \quad \sigma_{1}(x, \pi)=\sigma(x, \pi) \lambda_{\pi}^{-m / 2}, \quad \pi \in \widehat{G} \backslash\left\{1_{\widehat{G}}\right\} .
$$

Showing that $A_{1}$ is classical will imply that $A=A_{1}\left(\mathcal{L}_{G}+\chi\left(\mathcal{L}_{G}\right)\right)^{m / 2}$ is also classical, therefore this paragraph shows that we may assume $m=0$. 
Let us assume $m=0$. By the properties of invariance under translation (see Sect.4.2.5), it suffices to show that the operator $\exp ^{*} \chi_{1} A \chi_{2}$ is classical where $\chi_{1}, \chi_{2} \in C^{\infty}(G)$ have small supports near $e_{G}$ and identically equal to 1 on a small neighbourhood of $e_{G}$. Proceeding as in the proof of Lemma 4.5, the symbol $a$ of the operator $\exp ^{*} \chi_{1} A \chi_{2}$ is, up to a smoothing symbol, given by

$$
a(X, \xi)=\int_{Y \in \mathfrak{g}} \chi(Y) \kappa_{A, \exp _{G}(X)}\left(\exp _{G}(Y-X) \exp _{G}(X)\right) e^{i \xi \cdot Y}\left|\operatorname{jac}_{Y} \exp \right| d Y
$$

where $\chi \in C_{c}^{\infty}\left(\mathfrak{g}^{*}\right)$ is valued in $[0,1]$, supported near 0 and identically equal to 1 on a small neighbourhood of 0 . For $X \in \operatorname{supp} \chi$, the function $\Phi_{X}$ defined via

$$
\exp _{G}\left(\Phi_{X}(Y)\right)=\exp _{G}(Y-X) \exp _{G}(X), \quad Y \in \mathfrak{g}^{*},
$$

is smooth on $\mathfrak{g}^{*}$. As $D_{0} \Phi_{X}=\mathrm{I}$, it is a diffeomorphism between open neighbourhoods of 0 . The change of variable $Y^{\prime}=\Phi_{X}(Y)$ yields

$$
\begin{aligned}
a(X, \xi) & =b_{X}\left(\xi \circ \Phi_{X}^{-1}\right), \text { where } b^{(X)}(\xi) \\
& :=\int_{Y^{\prime} \in \mathfrak{g}} \chi\left(\Phi_{X}^{-1}\left(Y^{\prime}\right)\right) \kappa_{A, \exp _{G}(X)}\left(Y^{\prime}\right) e^{i \xi\left(Y^{\prime}\right)}\left|\operatorname{jac}_{Y^{\prime}} \exp \right| d Y^{\prime},
\end{aligned}
$$

viewing $\xi$ as an element of $\mathfrak{g}^{*}$. Setting

$$
b_{T}^{(X)}(w):=\left(\sigma\left(e^{X}, \pi\right) v, v\right)
$$

when $w \in \mathfrak{g}^{*}$ is an analytical integral weight for some $\pi \in \widehat{G}$ and maximal torus $T$, and where $v$ is a unit $w$-weight vector, we have

$$
b^{(X)}(w)-b_{T}^{(X)}(w)=\mathcal{F}_{G}\left\{\left(1-\chi_{X}\right) \kappa_{A, e^{X}}\right\}\left(\pi_{w}\right),
$$

where $\chi_{X}=\chi \circ \Phi_{X}^{-1} \circ \exp _{G}^{-1} \in C^{\infty}(G)$ is identically 1 in a neighbourhood of $e_{G}$. Setting $b_{T}^{(X)}(0)=0$, the function $b^{(X)}-b_{T}^{(X)}$ is therefore an invariant smoothing symbol on the lattice of analytical integral weight for the maximal torus $T$. Since $\sigma$ is 0 homogeneous, we have $b_{T}^{(X)}(k w)=b_{T}^{(X)}(w)$ and the function $b_{T}^{(X)}$ is a 0-homogeneous symbol on the lattice of analytical integral $T$-weights. By Proposition $3.3, b_{T}^{(X)}$ admits a unique smooth extension to $t^{*} \backslash\{0\}$ for which we keep the same notation. The uniqueness of the construction shows that we can define a function $b_{0}^{(X)}: \mathfrak{g}^{*} \rightarrow \mathbb{C}$ such that its restriction to the dual $t^{*}$ of the Lie algebra of any maximal torus $T$ coincides with $\left.b_{0}^{(X)}\right|_{\mathfrak{t}^{*}}=b_{T}^{(X)}$. Furthermore, $b_{0}^{(X)} \in C^{\infty}\left(\mathfrak{g}^{*} \backslash\{0\}\right)$ is 0-homogeneous.

We fix a function $\psi_{0} \in C^{\infty}(\mathbb{R})$ be such that $\psi_{0}(s)=0$ for $s \leq 1 / 2$ and $\psi_{0}(s)=1$ for $s \geq 1$. We define the smooth and bounded function $R$ on $\mathfrak{g}^{*}$ with

$$
R_{X}(\xi):=b^{(X)}(\xi)-b_{0}^{(X)}(\xi) \psi(\xi), \quad \psi(\xi):=\psi_{0}(|\xi|), \quad \xi \in \mathfrak{g}^{*}
$$


By construction, $R_{X}$ is an invariant symbol in $S^{0}$ on $\mathbb{R}^{n} \sim \mathfrak{g}^{*}$, therefore $\mathcal{F}_{\mathbb{R}^{n}}^{-1} R_{X}$ is Schwartz away from 0 . For any maximal torus $T$, the restriction of $R_{X}$ to the lattice of analytical integral weights of $T$ is smoothing, therefore it yields a smoothing symbol on the torus $T$ and its convolution kernel $\left.\mathcal{F}_{T}^{-1} R_{X}\right|_{T}$ is smooth near $e_{G} \in T$. This being true for any maximal torus $T$ implies that $\mathcal{F}_{\mathbb{R}^{n}}^{-1} R_{X}$ is Schwartz on $\mathfrak{g}$, so $R_{X} \in \mathcal{S}\left(\mathfrak{g}^{*}\right)$. One checks easily that the map $X \mapsto R_{X} \in \mathcal{S}\left(\mathfrak{g}^{*}\right)$ is smooth on a neighbourhood of 0 . As the symbol $a$ is given by

$$
a(X, \xi)=b_{0}^{(X)}\left(\xi \circ \Psi_{X}^{-1}\right) \psi\left(\xi \circ \Psi_{X}^{-1}\right)+R_{X}\left(\xi \circ \Psi_{X}^{-1}\right),
$$

it satisfies $a(X, \xi) \sim_{h} b_{0}^{(X)}\left(\xi \circ \Psi_{X}^{-1}\right)+0$. This concludes the proof.

The main ingredient for the reverse inclusion is the following lemma:

Lemma 4.11 Let $A \in \Psi_{c l}^{0}(G)$. As in Theorem 4.6, we identify its principal symbol with a smooth function $a_{0}: G \times\left(\mathfrak{g}^{*} \backslash\{0\}\right) \rightarrow \mathbb{C}$ which is 0 -homogeneous in the variable $\xi \in \mathfrak{g}^{*} \backslash\{0\}$.

Let $x \in G$ and let $\pi$ be an irreducible non-trivial representation of $G$. Fixing a maximal torus $T$, we define an endomorphism $M_{x, \pi}$ of $\mathcal{H}_{\pi}$ in the following way: all its coefficients with respect to an orthonormal basis of $\pi(T)$-eigenvectors of $\mathcal{H}_{\pi}$ vanish except (potentially) the diagonal ones corresponding to analytical integral weights $w$ where we have $\left(M_{x, \pi} v, v\right)_{\mathcal{H}_{\pi}}:=a_{0}(x, w)$. When $x$ runs over $G$ and $\pi$ runs over the set of irreducible non-trivial representation of $G$, the matrices $M_{x, \pi}$ together with $\sigma_{0}\left(x, 1_{\widehat{G}}\right)=0$ define a 0 -homogeneous symbol $\sigma_{0}$. Furthermore, $\sigma_{0} \in S^{0}(G)$ and $A-\mathrm{Op}\left(\sigma_{0}\right) \in \Psi_{c l}^{-1}(G)$.

Proof of Lemma 4.11 The considerations in Sects. 4.3 and 4.4 show that the symbol $\sigma_{0}$ is well-defined and 0-homogeneous. Let us show $\sigma_{0} \in S^{0}(G)$. We fix a maximal torus $T$. We will need the following observation: the highest weight theory implies that if $w_{1}, \ldots, w_{j}$ are $j$ analytical integral weights for representations $\pi_{1}, \ldots, \pi_{j} \in \widehat{G}$, then $w_{1}+\cdots+w_{j}$ is an analytical integral weights for the irreducible representation with highest weight (in any choice of ordering) in the decomposition of $\pi_{1} \otimes \cdots \otimes \pi_{j}$ into irreducibles, so we have:

$$
\begin{aligned}
& a_{0}\left(x, w_{1}+\cdots+w_{j}\right) \\
& \quad=\left(\sigma_{0}\left(x, \pi_{1} \otimes \cdots \otimes \pi_{j}\right) v_{1} \otimes \cdots \otimes v_{j}, v_{1} \otimes \cdots \otimes v_{j}\right)_{\mathcal{H}_{\pi_{1}} \otimes \cdots \otimes \mathcal{H}_{\pi_{j}}},
\end{aligned}
$$

where each vector $v_{k}$ is a unitary $w_{k}$-vector.

Let $\varphi, \pi \in \widehat{G}$. The endomorphsims $\sigma_{0}\left(x, 1_{\varphi} \otimes \pi\right)$ and $\sigma_{0}(x, \varphi \otimes \pi)$ of $\mathcal{H}_{\pi} \otimes \mathcal{H}_{\varphi}$ are diagonal when viewed in an orthogonal basis of vectors of the form $v_{\pi} \otimes v_{\varphi}$ where $v_{\pi}$ and $v_{\varphi}$ are eigenvectors for $\pi(\mathfrak{t})$ and $\varphi(\mathfrak{t})$ respectively. Furthermore, only the diagonal entries corresponding to analytical integral $w_{\pi}$ and $w_{\varphi}$ may be non-zero. Therefore this is also the case for $\Delta_{\varphi} \sigma_{0}(x, \pi)$ and we have by (4.4)

$$
\left(\Delta_{\varphi} \sigma_{0}(x, \pi) v_{\varphi} \otimes v_{\pi}, v_{\varphi} \otimes v_{\pi}\right)=a_{0}\left(x, w_{\varphi}+w_{\pi}\right)-a_{0}\left(x, w_{\pi}\right)
$$


This last expression is equal to $D_{\xi} a_{0}(x, \cdot)\left(w_{\pi}\right)$ for some $\xi \in\left[w_{\pi}, w_{\pi}+w_{\varphi}\right]$ by the mean value theorem. The homogeneity of $a_{0}$ then yield the estimate

$$
\left|\left(\Delta_{\varphi} \sigma_{0}(x, \pi) v_{\varphi} \otimes v_{\pi}, v_{\varphi} \otimes v_{\pi}\right)\right| \leq \mid w_{\varphi}\left\|\xi^{-1} \sup _{\left|\xi^{\prime}\right|=1}\right\| D_{\xi^{\prime}} a_{0}(x, \cdot) \| \lesssim a_{0}, \varphi\left\langle w_{\pi}\right\rangle^{-1}
$$

By [7, Lemma 3.8], $\left\langle w_{\pi}\right\rangle \asymp\left\langle\lambda_{\pi}\right\rangle^{1 / 2}$. Therefore, $\left\|\Delta_{\varphi} \sigma_{0}(x, \pi)\right\|_{\mathscr{L}\left(\mathcal{H}_{\pi \otimes \varphi}\right)} \lesssim a_{0}, \varphi$ $\left\langle\lambda_{\pi}\right\rangle^{-1 / 2}$.

More generally, we compute recursively for any symbol $\sigma$ independent of $x$ and $\varphi_{1}^{\prime}, \ldots, \varphi_{J}^{\prime}, \pi \in \operatorname{Rep}(G)$ :

$$
\begin{aligned}
\Delta_{\varphi_{1}^{\prime}} \ldots \Delta_{\varphi_{J}^{\prime}} \sigma(\pi)= & \sigma\left(x, \otimes_{k=1}^{J} \varphi_{k}^{\prime} \otimes \pi\right)-\sum_{j=1}^{J} \sigma\left(1_{\varphi_{j}^{\prime}} \otimes_{k \neq j} \varphi_{k}^{\prime} \otimes \pi\right) \\
& +\sum_{1 \leq j_{1}<j_{2} \leq J} \sigma\left(1_{\varphi_{j_{1}}^{\prime}} \otimes 1_{\varphi_{j_{2}}^{\prime}} \otimes_{k \neq j_{1}, j_{2}} \varphi_{k}^{\prime} \otimes \pi\right)+\ldots \\
& +(-1)^{J-1} \sum_{1 \leq j_{1}<j_{2} \leq J} \sigma\left(\varphi_{j_{1}}^{\prime} \otimes \varphi_{j_{2}}^{\prime} \otimes_{k \neq j_{1}, j_{2}} 1_{\varphi_{k}^{\prime}} \otimes \pi\right) \\
& +(-1)^{J} \sum_{j=1}^{J} \sigma\left(\varphi_{j}^{\prime} \otimes_{k \neq j} 1_{\varphi_{k}^{\prime}} \otimes \pi\right) .
\end{aligned}
$$

We now assume $\varphi_{1}^{\prime}, \ldots, \varphi_{J}^{\prime}, \pi$ irreducible. The endomorphsim $\Delta_{\varphi_{1}^{\prime}} \ldots \Delta_{\varphi_{J}^{\prime}} \sigma_{0}(x, \pi)$ is diagonal when viewed in an orthogonal basis of vectors which are the projections of of $v_{\pi} \otimes v_{\varphi_{1}^{\prime}} \otimes \cdots \otimes v_{\varphi_{J}^{\prime}}$ and only the diagonal entries corresponding to analytical integral weights $w_{\pi}$ and $w_{\varphi_{k}}$ may be non-zero. The formulae in (4.4) and (4.5) applied to symbols independent of $x$ on $\widehat{G}$ and also on the lattice of analytical integral weights yield:

$$
\begin{aligned}
& \left(\Delta_{\varphi_{1}^{\prime}} \ldots \Delta_{\varphi_{J}^{\prime}} \sigma_{0}(x, \pi) v_{\pi} \otimes v_{\varphi_{1}^{\prime}} \otimes \cdots \otimes v_{\varphi_{J}^{\prime}}, v_{\pi} \otimes v_{\varphi_{1}^{\prime}} \otimes \cdots \otimes v_{\varphi_{J}^{\prime}}\right) \\
& \quad=\Delta_{w_{\varphi_{1}^{\prime}}} \ldots \Delta_{w_{\varphi_{J}^{\prime}}} a_{0}\left(x, w_{\pi}\right)
\end{aligned}
$$

where $a(x, \cdot)$ is restricted to the lattice of analytical integral weights of $G$. Iteratively applying the mean value formula, we have

$$
\Delta_{w_{\varphi_{1}^{\prime}}} \ldots \Delta_{w_{\varphi_{J}^{\prime}}} a\left(x, w_{\pi}\right)=D_{\xi^{\prime}}^{J} a_{0}(x, \cdot)\left(w_{\varphi_{1}^{\prime}}, \ldots, w_{\varphi_{J}^{\prime}}\right)
$$

for some $\xi^{\prime}$ in a ball about $w_{\pi}$ and with radius $\sum_{j=1}^{J}\left|w_{\phi_{j}^{\prime}}\right|$. The homogeneity of $a_{0}$ then yield

$$
\left|D_{\xi^{\prime}}^{J} a_{0}(x, \cdot)\left(w_{\varphi_{1}^{\prime}}, \ldots, w_{\varphi_{J}^{\prime}}\right)\right| \leq\left|\xi^{\prime}\right|^{-J}\left|w_{\varphi_{1}^{\prime}}\right| \ldots\left|w_{\varphi_{J}^{\prime}}\right| \sup _{\left|\xi^{\prime}\right|=1}\left\|D_{\xi^{\prime}}^{J} a_{0}(x, \cdot)\right\| \lesssim\left\langle w_{\pi}\right\rangle^{-J}
$$


As $\left\langle w_{\pi}\right\rangle \asymp\left\langle\lambda_{\pi}\right\rangle^{1 / 2}$, we have obtained $\left\|\Delta_{\varphi_{1}^{\prime}} \ldots \Delta_{\varphi_{J}^{\prime}} \sigma_{0}(x, \pi)\right\|_{\mathscr{L}} \lesssim\left\langle\lambda_{\pi}\right\rangle^{-J / 2}$. This shows that the symbol $\sigma_{0}$ is in $S^{0}(G)$.

By Lemma 4.9, Op $\left(\sigma_{0}\right) \in \Psi_{c l}^{0}(G)$ so $A-\mathrm{Op}\left(\sigma_{0}\right)$ is in $\Psi_{c l}^{0}(G)$. By construction and Theorem 4.6, the principal symbol of $A-\mathrm{Op}\left(\sigma_{0}\right)$ is identically zero. This implies $A-\mathrm{Op}\left(\sigma_{0}\right) \in \Psi_{c l}^{-1}(G)$ and concludes the proof of Lemma 4.11.

Proof of Theorem 4.9 We have already noticed that Lemma 4.10 implies $\mathrm{Op}_{G}\left(S_{c l}^{m}(G)\right)$ $\subset \Psi_{c l}^{m}(G)$. Let us prove the reverse inclusion. Let $A \in \Psi_{c l}^{m}(G)$. Then $A\left(\mathrm{I}+\mathcal{L}_{G}\right)^{-\frac{m}{2}} \in$ $\Psi_{c l}^{0}(G)$ and we denote by $\sigma_{0}$ the 0 -homogeneous symbol associated with it in Lemma 4.11. We define $\sigma_{m}$ via $\sigma(x, \pi)=\lambda_{\pi}^{\frac{m}{2}} \sigma_{m}(x, \pi)$ for $\pi \in \widehat{G} \backslash\left\{1_{\widehat{G}}\right\}$, and $\sigma\left(x, 1_{\widehat{G}}\right)=0$. Then the symbol $\sigma_{m}$ is in $S^{m}(G)$ while the operator

$$
A-\mathrm{Op}\left(\sigma_{m}\right)=\left(A\left(\mathrm{I}+\mathcal{L}_{G}\right)^{-\frac{m}{2}}-\mathrm{Op}\left(\sigma_{0}\right)\right)\left(\mathrm{I}+\mathcal{L}_{G}\right)^{\frac{m}{2}} \bmod \Psi^{-\infty},
$$

is in $\Psi_{c l}^{m-1}(G)$. Recursively, we obtain a poly-homogeneous expansion $\sum_{j \in \mathbb{N}_{0}} \sigma_{m-j}$ for $\sigma=\mathrm{Op}_{G}^{-1} A$ and this shows $\sigma \in S_{c l}^{m}(G)$. We have obtained the reverse inclusion $\Psi_{c l}^{m}(G) \subset \mathrm{Op}_{\mathbb{T}^{n}}\left(S_{c l}^{m}(G)\right)$ and this concludes the proof of Theorem 4.9.

\subsection{Non-commutative residue and canonical trace on $G$}

Here, we generalise Corollary 3.6 to the case of a compact Lie group which may not be commutative, i.e. may not be the torus. Applying Theorem 2.5 and Proposition 2.9 to the Laplace operator $\mathcal{L}_{G}$, we obtain readily the following result.

Proposition 4.12 Let $\sigma \in S_{c l}^{m}(G)$ with $m \in \mathbb{C}$. We set $A:=\operatorname{Op}_{G}(G)$.

(2) Let $\eta \in C_{c}^{\infty}(0, \infty)$. Then we have an expansion as $t \rightarrow 0^{+}$:

$$
\operatorname{tr}\left(A \eta\left(\mathcal{L}_{G}\right)\right)=\sum_{\pi \in \widehat{G}} d_{\pi} \eta\left(t \lambda_{\pi}\right) \operatorname{tr}(\sigma(x, \pi)) \sim c_{m+n} t^{-\frac{m+n}{m_{0}}}+c_{m-n-1} t^{-\frac{m+n-1}{m_{0}}}+\ldots
$$

The constants $c_{m+n-j}$ are of the form $c_{m+n-j}=c_{m+n-j}^{(\sigma)} c_{m+n-j}^{(\eta)}$ where $c_{m+n-j}^{(\sigma)}$ depends only on the homogeneous expansion of $\sigma$ and

$$
c_{m+n-j}^{(\eta)}:=\frac{1}{2} \int_{u=0}^{+\infty} \eta(u) u^{\frac{m-j+n}{2}} \frac{d u}{u} .
$$

If $m \in \mathbb{Z}_{n}$ then $c_{0}=c_{0}^{(\sigma)} c_{0}^{(\eta)}$ with

$$
c_{0}^{(\sigma)}=\operatorname{res} A, \quad \text { and } \quad c_{0}^{(\eta)}=\frac{1}{2} \int_{0}^{+\infty} \eta(u) \frac{d u}{u} .
$$

(3) Here, $\Re m \geq-n$ with $m \notin \mathbb{Z}$. Let $\eta \in \mathcal{M}^{m_{\eta}}(\mathbb{R})$ (see Definition 2.4) with $\Re m+$ $m_{\eta} m_{0}<-n$. Then the operator $A \eta(t \Delta)$ is traceclass for all $t \in \mathbb{R}$ and the trace 
admits the following expansion as $t \rightarrow 0^{+}$,

$$
\begin{aligned}
\operatorname{tr}\left(A \eta\left(\mathcal{L}_{G}\right)\right)= & \sum_{\pi \in \widehat{G}} d_{\pi} \eta\left(t \lambda_{\pi}\right) \operatorname{tr}(\sigma(x, \pi))=\eta(0) \operatorname{TR}(A) \\
& +\sum_{j=0}^{N-1} c_{m+n-j}^{\prime} t^{\frac{-m-n+j}{2}}+o(1),
\end{aligned}
$$

where $N \in \mathbb{N}$ is the smallest non-negative integer such that $N \geq 2 m_{\eta}+m_{1}+n$. The constants $c_{m+n-j}^{\prime}$ are of the form $c_{m+n-j}^{\prime}={c^{\prime}}_{m+n-j}^{(\sigma)}{c^{\prime}(\eta)}_{m+n-j}$ where $c_{m+n-j}^{(\sigma)}$ depends only on the poly-homogeneous expansion of $\sigma$ and

$$
c_{m+n-j}^{\prime(\eta)}:=\frac{1}{2} \int_{u=0}^{+\infty} \eta(u) u^{\frac{m-j+n}{2}} \frac{d u}{u} .
$$

Open Access This article is distributed under the terms of the Creative Commons Attribution 4.0 International License (http://creativecommons.org/licenses/by/4.0/), which permits unrestricted use, distribution, and reproduction in any medium, provided you give appropriate credit to the original author(s) and the source, provide a link to the Creative Commons license, and indicate if changes were made.

\section{References}

1. Agranovič, M.S.: Spectral properties of elliptic pseudodifferential operators on a closed curve. Funkt. Anal. Prilozhen. 13(4), 54-56 (1979)

2. Agranovič, M.S.: Elliptic pseudodifferential operators on a closed curve. Trudy Mosk. Mat. Obshch. 47(246), 22-67 (1984)

3. Connes, A.: Non-commutative Geometry. Academic Press Inc, San Diego (1994)

4. Dixmier, J.: von, Neumann algebras, 27, Translated from the Second French Edition by F. NorthHolland Publishing Co., Amsterdam-New York, Jellett (1981)

5. Fedosov, B.V., Golse, F., Leichtnam, E., Schrohe, E.: The non-commutative residue for manifolds with boundary. J. Funct. Anal. 142(1), 1-31 (1996)

6. Fischer, V.: Real trace expansion. preprint at arXiv

7. Fischer, V.: Differential structure on the dual of a compact Lie group. preprint at arXiv:1610.06348

8. Fischer, V.: Intrinsic pseudodifferential calculi on any compact Lie group. J. Funct. Anal. 268(11), 3404-3477 (2015)

9. Grubb, G., Schrohe, E.: Traces and quasi-traces on the Boutet de Monvel algebra. Ann. Inst. Fourier (Grenoble) 54(5), 1641-1696 (2004). xvii, xxii

10. Grubb, G.: A resolvent approach to traces and zeta Laurent expansions, spectral geometry of manifolds with boundary and decomposition of manifolds. Contemp. Math. 366, 67-93 (2005). Amer. Math. Soc., Providence, RI,

11. Grubb, G., Seeley, R.T.: Weakly parametric pseudodifferential operators and Atiyah-Patodi-Singer boundary problems. Invent. Math. 121(3), 481-529 (1995)

12. Guillemin, V.: A new proof of Weyl's formula on the asymptotic distribution of eigenvalues. Adv. Math. 55(2), 131-160 (1985). Advances in Mathematics

13. Hall, B.: Lie Groups, Lie Algebras, and Representations. Graduate Texts in Mathematics, 222, 2nd edn. Springer, Cham (2015)

14. Helgason, S.: Groups and Geometric Analysis. Mathematical Surveys and Monographs, vol. 83. American Mathematical Society, Providence (2000). Reprint of the 1984 original

15. Hörmander, L.: The Analysis of Linear Partial Differential Operators. I, Classics in Mathematics. Springer, Berlin (1983) 
16. Knapp, A.: Lie Groups Beyond an Introduction. Progress in Mathematics, vol. 140. Birkhäuser Boston Inc., Boston MA (1996)

17. Kontsevich, M., Vishik, S.: Geometry of determinants of elliptic operators. In: Functional Analysis on the Eve of the 21st Century, Vol. 1 (New Brunswick, NJ, 1993), Progr. Math., vol. 131, pp. 173-197. Birkhäuser Boston (1995)

18. Lesch, M.: On the non-commutative residue for pseudodifferential operators with logpolyhomogeneous symbols. Ann. Glob. Anal. Geom. 17(2), 151-187 (1999)

19. Lesch, M.: Pseudodifferential operators and regularized traces. In: Motives, Quantum Field Theory, and Pseudodifferential Operators. Clay Math. Proc. vol. 12, pp. 37-72. Amer. Math. Soc., Providence, RI (2010)

20. Lévy, C., Jiménez, N.C., Paycha, S.: The canonical trace and the non-commutative residue on the non-commutative torus. Trans. Am. Math. Soc. 368(2), 1051-1095 (2016)

21. McLean, William: Local and global descriptions of periodic pseudodifferential operators. Math. Nachr. 150, 151-161 (1991)

22. Melo, S.T.: Characterizations of pseudodifferential operators on the circle. Proc. Am. Math. Soc. 125(5), 1407-1412 (1997)

23. Nicola, F., Rodino, L.: Global pseudo-differential calculus on Euclidean spaces, Pseudo-Differential Operators. Theory and Applications, vol. 4. Birkhäuser Verlag, Basel (2010)

24. Okikiolu, K.: Critical metrics for the determinant of the Laplacian in odd dimensions. Ann. Math. (2) 153(2), 471-531 (2001)

25. Paycha, S.: Regularised Integrals, Sums and Traces, University Lecture Series, 59. American Mathematical Society, Providence, RI, An analytic point of view (2012)

26. Pietsch, A.: Traces and residues of pseudo-differential operators on the torus. Integral Equ Oper Theory 83(1), 1-23 (2015)

27. Ruzhansky, M., Turunen, V.: Pseudodifferential Operators and Symmetries, Pseudodifferential Operators. Theory and Applications 2, Background analysis and advanced topics. Birkhäuser Verlag, Basel (2010)

28. Ruzhansky, M., Turunen, V., Wirth, J.: Hörmander class of pseudodifferential operators on compact Lie groups and global hypoellipticity. J. Fourier Anal. Appl. 20(3), 476-499 (2014)

29. Schrohe, E.: Wodzicki's noncommutative residue and traces for operator algebras on manifolds with conical singularities. In: Microlocal Analysis and Spectral Theory (Lucca, 1996), NATO Adv. Sci. Inst. Ser. C Math. Phys. Sci., vol. 490, pp. 227-250, Kluwer Acad. Publ., Dordrecht (1997)

30. Scott, S.: Traces and Determinants of Pseudodifferential Operators. Oxford Mathematical Monographs. Oxford University Press, Oxford (2010)

31. Seeley, R.T.: Complex powers of an elliptic operator. In: Singular Integrals (Proc. Sympos. Pure Math., Chicago, Ill., pp. 288-307, p. 1967. Amer. Math. Soc, Providence, RI (1966)

32. Shubin, M.: Pseudodifferential Operators and Spectral Theory. Translated from the 1978 Russian original by Stig I. Andersson, 2nd edn. Springer-Verlag, Berlin (2001)

33. Taylor, M.: Pseudodifferential Operators. Princeton Mathematical Series, vol. 34. Princeton University Press, Princeton (1981)

34. Taylor, M.: Noncommutative Harmonic Analysis. Mathematical Surveys and Monographs, vol. 22. American Mathematical Society, Providence, RI (1986)

35. Turunen, V., Vainikko, G.: On symbol analysis of periodic pseudodifferential operators. Z. Anal. Anwend. 17(1), 9-22 (1998)

36. Wodzicki, M.: Local invariants of spectral asymmetry. Invent. Math. 75(1), 143-177 (1984)

37. Wodzicki, M.: Noncommutative residue. I. Fundamentals. In: K-Theory, Arithmetic and Geometry (Moscow, 1984-1986), Lecture Notes in Math., vol. 1289, pp. 320-399. Springer, Berlin (1987)

Publisher's Note Springer Nature remains neutral with regard to jurisdictional claims in published maps and institutional affiliations. 